\title{
Distortion-Driven Video Streaming over Multihop Wireless Networks with Path Diversity
}

\author{
Xiaolin Tong, Yiannis Andreopoulos, Member, IEEE, and \\ Mihaela van der Schaar, Senior Member, IEEE
}

\begin{abstract}
Multihop networks provide a flexible infrastructure that is based on a mixture of existing access points and stations interconnected via wireless links. These networks present some unique challenges for video streaming applications due to the inherent infrastructure unreliability. In this paper, we address the problem of robust video streaming in multihop networks by relying on delayconstrained and distortion-aware scheduling, path diversity, and retransmission of important video packets over multiple links to maximize the received video quality at the destination node. To provide an analytical study of this streaming problem, we focus on an elementary multihop network topology that enables path diversity, which we term "elementary cell." Our analysis is considering several cross-layer parameters at the physical and medium access control (MAC) layers, as well as application-layer parameters such as the expected distortion reduction of each video packet and the packet scheduling via an overlay network infrastructure. In addition, we study the optimal deployment of path diversity in order to cope with link failures. The analysis is validated in each case by simulation results with the elementary cell topology, as well as with a larger multihop network topology. Based on the derived results, we are able to establish the benefits of using path diversity in video streaming over multihop networks, as well as to identify the cases where path diversity does not lead to performance improvements.
\end{abstract}

Index Terms-Wireless systems, multimedia information systems, video, multihop networks, distortion-aware path diversity, cross-layer design.

\section{INTRODUCTION}

$\mathrm{V}$ IDEO streaming over multihop wireless networks has recently attracted significant attention [1], [2]. This is due to the fact that the interconnection of existing highspeed IEEE 802.11 wireless LANs in residential or office environments into one multihop topology [3] and recent quality-of-service (QoS)-enabled Medium Access Control (MAC) protocols [4] provides new opportunities for such delay-constrained high-bandwidth applications [5]. However, for the foreseeable future, commercial wireless multihop networks will be built upon unreliable nodes (wireless access points or stations) that are connected by wireless links with highly varying channel conditions [3]. Therefore, even under QoS guarantees for the MAC layer, robust video streaming applications must still be able to tolerate link failures or deep link fading, which may occasionally occur due to node mobility or the unwillingness of the user to share node resources in the multihop topology [3]. Thus, a variety of protection schemes deployed in the different layers of the protocol stack should be explored and evaluated under different scenarios.

- X. Tong is with the Corp RED Department, Qualcomm Inc., 5775 Morehouse Drive, San Diego, CA 92121. E-mail: xltong@ee.ucla.edu.

- Y. Andreopoulos is with the Department of Electronic Engineering, Queen Mary University of London, Mile End Road, London, E1 4NS, UK. E-mail: yiannis.a@elec.qmul.ac.uk.

- M. van der Schaar is with the Department of Electrical Engineering, University of California Los Angeles, 66-147E Engineering IV Building, 420 Westwood Plaza, Los Angeles, CA, 90095-1594.

E-mail: mihaela@ee.ucla.edu.

Manuscript received 31 Mar. 2006; revised 3 Mar. 2007; accepted 21 Mar. 2007; published online 2 Apr. 2007.

For information on obtaining reprints of this article, please send e-mail to: tmc@computer.org, and reference IEEECS Log Number TMC-0093-0306.

Digital Object Identifier no. 10.1109/TMC.2007.1063.

\subsection{Related Work}

From the application-layer perspective, path diversity emerged as an efficient mechanism for providing robustness in video communications [2], [6], [7], [8], especially when node or path failures occur [9]. Path diversity involves the adaptive selection of two or more network paths for the transmission of each video packet using an overlay network infrastructure [14] at the application layer. Although previous work applies path diversity with nonscalable multiple-description coding (for example, see [2] and [7]), we utilize path diversity and scalable video coding [16]. Our solution has low complexity and provides the opportunity to introduce redundancy postencoding at the packet level. Hence, source coding efficiency is not sacrificed for the sake of robustness and redundancy can be added on the fly during the streaming process. Moreover, if additional complexity is permitted, this approach can be combined with forward error correction mechanisms for additional efficiency [10]. Although prior work showed the benefits of path diversity under various transmission scenarios, its merits under realistic cross-layer protection mechanisms (and, in particular, for the case of wireless multihop networks) are largely unknown. Given the fact that crosslayer frameworks for real-time video streaming over ad hoc wireless networks also exhibit significant improvements in robustness over single-layer optimization at the application layer [1], [5], [18], we believe that the investigation of path diversity for video streaming under a realistic cross-layer optimization framework is a significant open challenge.

\subsection{Proposed Solutions for Multihop Video Streaming with Path Diversity}

In this work, we address this problem by utilizing distortion-optimal packet prioritization at the application 
layer (derived by state-of-the-art scalable video coding [16]) in order to make optimal packet ordering and optimal retransmissions at the MAC analytically tractable [17]. In this paper, an operational distortion model for scalable coded bitstreams is proposed (Section 2.2) in order to prioritize the packet transmission of each video flow according to the importance in the decoded video quality.

Given this strict prioritization for the packets of each video flow, our solution optimally decides among several choices for path diversity, the number of packet retransmissions at the MAC, and the optimal link adaptation strategy (that is, modulation scheme [19], [20]) at the physical (PHY) layer. Based on the knowledge of local network conditions, we select an elementary network topology that suits our multihop network simulation and analytically derive the optimal streaming parameters for the various layers of the protocol stack. The proposed approach is then generalized across the entire multihop topology in order to obtain locally optimal streaming solutions. Based on the proposed framework, we are able to address several open questions:

1. whether the optimized application-layer path diversity provides additional benefits over conventional retransmission schemes at the MAC and link adaptation at the PHY in the scenario that no link failure happens,

2. how many packets (if any) should use path diversity in the case of link failures, and

3. how close a cross-layer optimized approach with path diversity is to the performance limit, which assumes exact knowledge of the instantaneous multihop network conditions and the link-failure occurrences and adaptively chooses a network path based on this knowledge.

\subsection{Network Topology, Routing, and MAC-Layer Assumptions}

We assume that link failures occur due to sudden bursts of severe interference, for example, due to node mobility. In addition, we assume that a predetermined transmission opportunity interval is reserved for each video flow on the various network nodes, following the concepts of Hybrid Coordination Function (HCF) Controlled Channel Access (HCCA) mechanisms of IEEE 802.11e [4]. We envisage that such a reservation mechanism can be deployed and controlled either by a central coordinator in the multihop network [11] or by a distributed solution using an overlay network infrastructure [12], [13]. In this way, the available resources can be determined based on the video flows sharing the network and their QoS requirements. In particular, we assume that the mesh network topology is fixed over the duration of the video session (barring failures) and that, prior to transmission, each application (video flow) reserves a predetermined transmission opportunity interval, where contention-free access to the medium is provided. ${ }^{1}$ This reservation can be determined based on the number of flows sharing the network. Although the design of such a coordination and reservation system is an

1. Existing IEEE standards [4] already support such QoS mechanisms, which, barring interference and environment noise, provide guaranteed transmission time for each admitted application (video flow). important problem and it affects our results, recent work [11], [12], [13] showed that the scheduling of multiple flows in the context of a mesh topology can be done such that the average rate for every flow is satisfied and the interference to neighboring nodes is minimized [11]. In our paper, we adopt a similar overlay network solution that operates at the application layer, spanning the various IEEE 802.11e wireless cells. Once the available network infrastructure to the video streaming session has been established, we assume that an overlay network topology can convey (in frequent intervals) information about the expected bit error rate (BER), the queuing delay for each link, and the guaranteed bandwidth under the dynamically changing modulation at the PHY. This overlay infrastructure can also minimize the probability of additional delays and link failures by adaptively routing the video packets. Several examples of such application-layer overlay networks have been proposed in the literature [12], [14].

Finally, we assume that the application layer of every node in the multihop network relies on an overlay infrastructure [14] that can

1. route video packets via selected links to the destination node and

2. convey information about the expected BER, the queuing delay for each link, and the guaranteed bandwidth under link adaptation from the neighboring nodes in the network topology [5].

\subsection{Paper Organization}

The paper is organized as follows: Section 2 defines the scenarios examined in this work and provides the necessary definitions and formulations for the expected bandwidth, transmission error rate, and the expected delay for streaming under various network paths. Section 3 presents the cross-layer optimization problem for the different cases that potentially involve path diversity and link failures and analyzes the performance of the proposed scheme under each scenario. Section 4 presents simulation results and validation for the theoretical study. Our conclusions are presented in Section 5.

\section{Problem Formulation}

\subsection{Topology Specification and Parameter Definitions}

The wireless multihop network topology between the video server (sender) and the client (receiver) can be expressed by a connectivity structure $\mathcal{P}$ with $M$ paths, that is, $\mathcal{P}=\left\{\mathbf{p}_{1}, \ldots, \mathbf{p}_{M}\right\}$. Each path $\mathbf{p}_{i}(1 \leq i \leq M)$ consists of

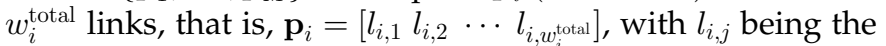
$j$ th link of path $\mathbf{p}_{i}$. An example is given in Fig. 1, where two possible paths from sender $\mathrm{n} 0$ to receiver $\mathrm{n} 6$ are highlighted. We assume that all utilized paths from sender to receiver are determined beforehand, for example, during the video-session configuration setup, following conventional route discovery algorithms. Assuming that a timereservation mechanism following the IEEE 802.11e principles is deployed at the MAC layer, the transmission opportunity duration is preestablished for each link $l_{i, j}$ $\left(1 \leq i \leq M, 1 \leq j \leq w_{i}^{\text {total }}\right)$ based on a negotiation scheme, 


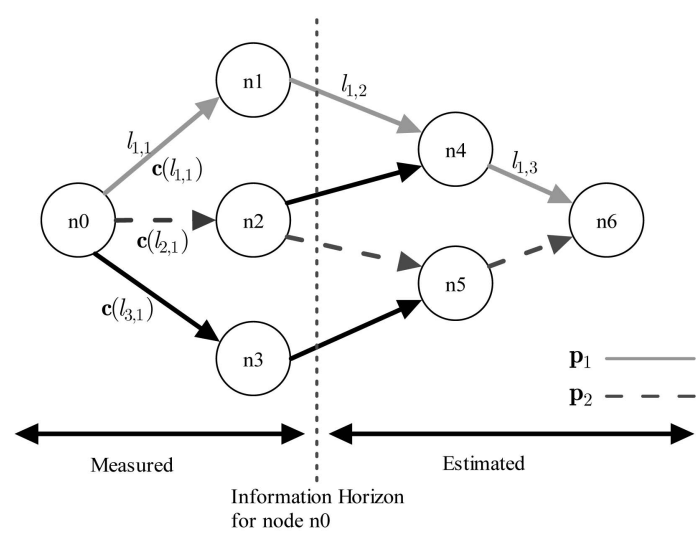

Fig. 1. A topology example illustrating two examples of paths between the sender and receiver nodes, as well as the link state vectors $c\left(l_{i, 1}\right)$, $i=\{1, \ldots, 3\}$. Network information before the indicated "horizon" is actively monitored (measured) by the sender node, whereas information at the horizon and beyond is estimated.

for example, via node resource exchange strategies moderated by a central coordinator. Thereafter, the guaranteed bandwidth $g\left(l_{i, j}\right)$ of link $l_{i, j}$ can be determined based on the allocated transmission opportunity duration [5]. In addition, the experienced signal-to-interference-noise ratio (SINR) $s\left(l_{i, j}\right)$ can also be derived following previous channel estimation approaches [19], [20]. We augment the SINR estimation at the PHY layer with the link-failure probability $f\left(l_{i, j}\right)$, which represents the expected failure probability for the duration of the video streaming session. Even though link failures may be short-lived, it is safe to assume that all video packets on a failed link are lost during the occurrence of a failure due to the stringent delays of typical video streaming applications. In total, we define the state vector for each link $l_{i, j}$ as

$$
\mathbf{c}\left(l_{i, j}\right)=\left[g\left(l_{i, j}\right) \quad s\left(l_{i, j}\right) \quad f\left(l_{i, j}\right)\right] .
$$

In our work, we assume that each node can actively monitor the state vector of each outgoing link. However, link state information beyond the immediate neighborhood can only be estimated. This follows the notion of network "horizons" introduced in our recent work [5]. An example of this principle is pictorially shown in Fig. 1 for the sender node n0. Based on this assumption, all network conditions (for example, available bandwidth or packet loss rate) beyond the links of the sender node are expressed as statistical expectations, and their exact value during the network simulation is unknown.

\subsection{Video Source Characteristics}

At the application layer, the video stream to be transmitted is encapsulated in a sequence of video packets. Each video packet $v$ is characterized by its deadline $d_{v}^{\text {deadline }}$, the packet size $L_{v}$, and the induced distortion reduction $\Delta_{v}$ at the video decoder. These parameters are communicated from the application to the MAC layer following a multitrack hint specification [15]. The packet deadline ensures that each node will forward the packet only if it is still useful atthe decoder; otherwise, the video packet is dropped. Thepacket size is useful during the transmission delay estimation and the error probability calculations, as it will be shown in Section 2.3. Finally, the distortion reduction is a utility metric that enforces appropriate prioritization strategies for each video packet for MAC-layer scheduling and retransmission. Following recent state-of-the-art scalable video coding schemes [16], an additive distortion model can be used to associate distortion reductions to the video packets belonging to a Group of Pictures (GOP) with the same deadline. ${ }^{2}$ Packets in each GOP are ordered by their distortion reduction $\Delta_{v}, v=1, \ldots, N_{G}$, that is, $\Delta_{1}>\Delta_{2}>\ldots>\Delta_{N_{G}}$, where $N_{G}$ is the total number of packets in GOP $G$. In this paper, we model the distortion reduction of a packet $v$ in each GOP by

$$
\Delta_{v}=\alpha \cdot \rho^{v} \quad \rho \in(0,1),
$$

where $\alpha$ and $\rho$ are modeling parameters that depend on sequence characteristics and the encoding parameters. As it will be illustrated in the experimental part of the paper, this model can approximate the measured distortion reduction for a variety of video sequences encoded by a wavelet video encoder by the appropriate selection of $\alpha$ and $\rho$ for each GOP. Notice that the strict distortion reduction prioritization expressed by the monotonic function of (2) enables the exact analysis of the expected MAC operation under simple network topologies since packets are encapsulated in MAC Service Data Units (MSDUs) in a predetermined order based on their distortion reduction. This is an important aspect of our work since, unlike related work with nonscalable coding [2], [7], [21], our preestablished prioritization mechanism leads to a very low-complexity solution. This fact, combined with the individual MSDU acknowledgments received at the MAC layer, provides a tractable solution for determining several optimal cross-layer parameters during the real-time video streaming session.

\subsection{Expected Transmission Error Probability and Delay Estimation at the MAC Layer}

Based on the above definitions, the MAC-layer probability of error for packet $v$ of size $L_{v}$ bits over link $l_{i, j}$ can be given as

$$
e_{l_{i, j}}\left(L_{v}\right)=1-\left(1-e\left(l_{i, j}\right)\right)^{L_{v}}
$$

where $e\left(l_{i, j}\right)$ expresses the MAC-layer BER of link $l_{i, j}$, which can be estimated based on the chosen modulation $m\left(l_{i, j}\right)$ and the observed SINR $s\left(l_{i, j}\right)$ [19], [20]. Similarly, the probability of error for one transmission over path $\mathbf{p}_{i}$ is

$$
e_{\mathbf{p}_{i}}\left(L_{v}\right)=1-\prod_{j=1}^{w_{i}^{\text {total }}}\left(1-e_{i_{i, j}}\left(L_{v}\right)\right)=1-\prod_{j=1}^{w_{i}^{\text {total }}}\left(\left(1-e\left(l_{i, j}\right)\right)^{L_{v}}\right) .
$$

Let $N_{\mathbf{p}_{i}}^{\max }$ be the maximum number of retransmissions for packet $v$ over path $\mathbf{p}_{i}$. The average number of transmissions over path $\mathbf{p}_{i}$ until the packet is successfully transmitted or the retransmission limit is reached can then be evaluated as follows [18]:

2. For simplicity and without loss of generality, we assume that delay deadlines are associated with the GOP structure of the video coder. Smaller delays can be accommodated following the notion of subflows [5]. 


$$
\begin{aligned}
N_{\mathbf{p}_{i}}^{\operatorname{mean}}\left(N_{\mathbf{p}_{i}}^{\max }\right)= & \sum_{j=1}^{N_{\mathbf{p}_{i}}^{\max }+1}\left(j \cdot\left[e_{\mathbf{p}_{i}}\left(L_{v}\right)\right]^{j-1}\left[1-e_{\mathbf{p}_{i}}\left(L_{v}\right)\right]\right) \\
& +\left[N_{\mathbf{p}_{i}}^{\max }+1\right] \cdot\left[e_{\mathbf{p}_{i}}\left(L_{v}\right)\right]^{N_{\mathbf{p}_{i}}^{\max }+1} \\
& =\frac{1-\left[e_{\mathbf{p}_{i}}\left(L_{v}\right)\right]^{N_{\mathbf{p}_{i}}^{\max }+1}}{1-e_{\mathbf{p}_{i}}\left(L_{v}\right)} .
\end{aligned}
$$

Thus, the average end-to-end delay of a packet with size $L_{v}$ over $\mathbf{p}_{i}$ is

$$
\begin{aligned}
& d_{\mathbf{p}_{i}}\left(L_{v}, N_{\mathbf{p}_{i}}^{\text {mean }}\left(N_{\mathbf{p}_{i}}^{\max }\right)\right)= \\
& N_{\mathbf{p}_{i}}^{\text {mean }}\left(N_{\mathbf{p}_{i}}^{\max }\right) \cdot \sum_{j=1}^{w_{i}^{\text {total }}}\left(\frac{L_{v}}{g\left(l_{i, j}\right)}+T_{\text {overhead }}\right)+\sum_{j=1}^{w_{i}^{\text {total }}} d_{\text {queue }}\left(l_{i, j}\right),
\end{aligned}
$$

where $T_{\text {overhead }}$ accounts for the delay and protocol overheads involved in the packet transmission. The last equation derives the end-to-end delay estimate by joining all links of path $\mathbf{p}_{i}$ via the summation terms, thereby forming a "virtual" link from the sender to the receiver node in the multihop network. We follow this approach since the maximum number of retransmissions $\mathrm{T}_{\mathbf{p}_{i}}^{\max }$ required on each path $\mathbf{p}_{i}$ can only be defined from end to end based on the maximum permissible delay from the sender node to the receiver. We remark that, in our experiments, the retransmission limit for any part of a path or even for one link $l_{i, j}$ is set equal to $\mathrm{T}_{\mathbf{p}_{i}}^{\max }$ since, in principle, all possible retransmissions (until the MSDU expires due to delay violation) could occur at an individual link. Following the analysis of (4)-(7), it is straightforward to define the average MSDU transmissions and the expected delay for subpaths that include only a subset of links or even for an individual link. This will be proven to be very useful for some of the derivations.

With the knowledge of the packets scheduled for transmission over a certain link $l_{i, j}$, denoted by $\mathbf{v}_{\text {queue }}\left(l_{i, j}\right)$, we can calculate the queuing delay for that link. For each packet $w$ in $\mathbf{v}_{\text {queue }}\left(l_{i, j}\right)$, the product of the expected number of transmissions of the packet (given by (6)) with the transmission duration gives the average delay to transmit the packet under the particular network conditions (quantified by bandwidth $g\left(l_{i, j}\right)$ and packet loss rate $e_{\mathbf{p}_{i}}\left(L_{w}\right)$ from (4)). The result is

$$
d_{\text {queue }}\left(l_{i, j}\right)=\sum_{\forall w \in \mathbf{v}_{\text {queue }}\left(l_{i, j}\right)} N_{\mathbf{p}_{i}}^{\text {mean }}\left(N_{\mathbf{p}_{i}}^{\max }\right) \cdot\left(\frac{L_{w}}{g\left(l_{i, j}\right)}+T_{\text {overhead }}\right) .
$$

\subsection{Elementary Cells in Multihop Wireless Networks-A Divide and Conquer Strategy}

Although the estimations of packet error probability and delay of the previous section may be accurate for the outgoing links of each node, the uncertainty introduced for remote links in the multihop topology, combined with the approximations ${ }^{3}$ necessary for making practical predictions based on (5)-(8), makes the analysis of the full topology very

3. For example, one has to assume a constant (nominal) packet size, known link and path error probability, and so forth.

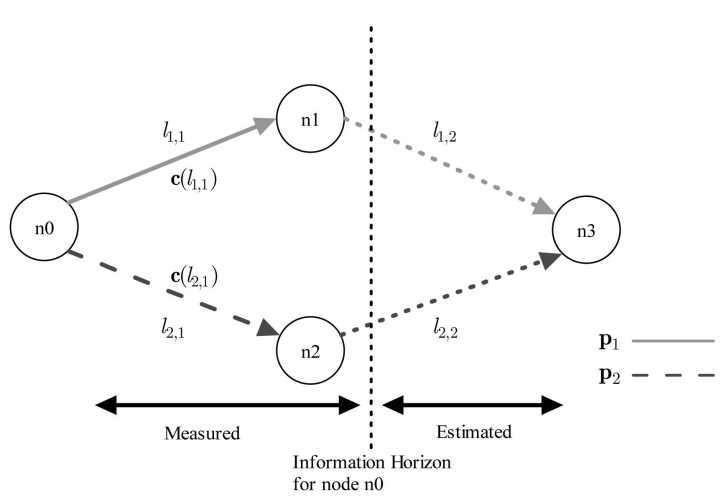

(a)

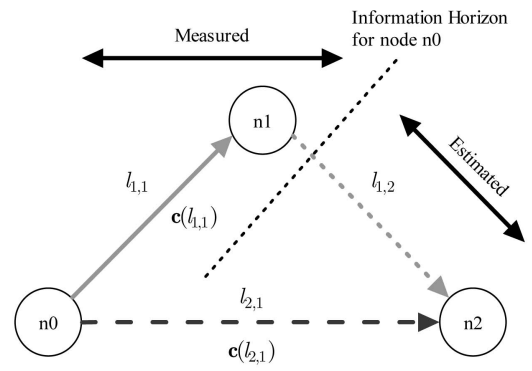

(b)

Fig. 2. Two examples of multihop wireless network elementary cells.

difficult. In fact, our previous research has already shown that such estimation-based schemes may lead to significant gaps in performance in comparison to the end-to-end optimization of video streaming under full knowledge of the network conditions. On the other hand, optimization under the knowledge of the local network status performed reasonably well [5], even when compared to end-to-end optimization. Following this lead, in this paper, we extend the concept of limited-horizon video streaming optimization for multihop networks to optimization within a multihop wireless network "elementary cell."

Pictorial examples of such elementary cells are given in Fig. 2. In the elementary cell in Fig. 2a, node n0 (source node) has only two state vectors, $\mathbf{c}\left(l_{1,1}\right)$ and $\mathbf{c}\left(l_{2,1}\right)$. Fig. $2 \mathrm{~b}$ can be considered as a special case of Fig. 2a in which node $\mathrm{n} 2$ and $\mathrm{n} 3$ (destination node) are collapsed into one node. Based on an overlay network infrastructure at the application layer [14], each source node of an elementary cell in the overall multihop topology operates assuming that the video sender and receiver are located in the starting and terminating nodes of its cell and video packets can be routed from the sender to the receiver node following a specific path. In this way, path diversity within an elementary cell becomes feasible; also, it is significantly simpler in comparison to the direct analysis of path diversity over large multihop networks. Finally, this approach allows for an analytical study of the merits and disadvantages of path diversity under realistic conditions for the MAC and PHY layers since it corresponds to the basic scenario where two nonoverlapping paths (with uncertain conditions) are available in the wireless multihop network for the video flow.

When considered within the overall multihop network topology, a certain network node may be the origin of more 


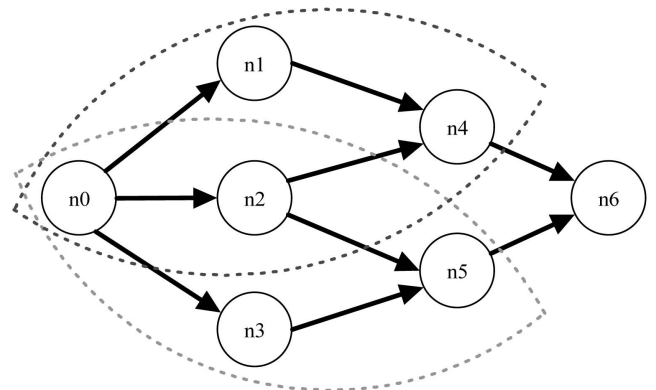

(a)

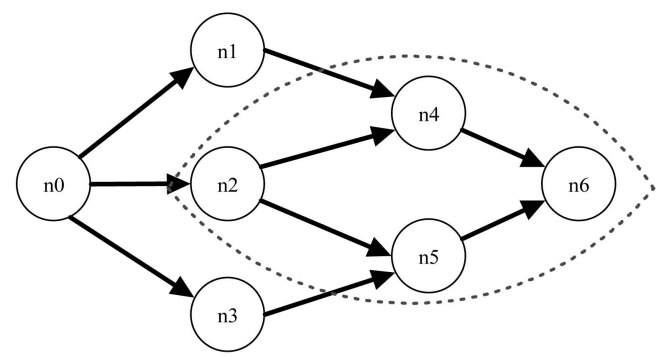

(b)

Fig. 3. (a) The two initial elementary cells emerging from no (source node). (b) The subsequent network cell from the intermediate network node $\mathrm{n} 2$ to the final destination node n6. Notice that, in the case indicated in (b), node $\mathrm{n} 2$ is the source node for the elementary cell.

than one elementary cell. Moreover, intermediate nodes of an elementary cell may also be the source nodes of subsequent cells. This is illustrated in the example in Fig. 3. In this way, wireless multihop network topologies can be decomposed in elementary cells introduced in Fig. 2a. This also covers the cases where more than two nodes are connected with a source node, as shown for node $\mathrm{n} 0$ in Fig. 3. In the remainder of the paper, the term "elementary cell" refers to cells of the type introduced in Fig. 2a and displayed in the examples in Fig. 3.

\section{Path Diversity Study in Elementary Cells}

This section proposes cross-layer optimized video streaming with path diversity in the proposed framework of a wireless multihop elementary cell topology. Although we focus our attention on the multihop elementary cell in Fig. 2a, our analysis is applicable in a broad range of similar configurations. Sections 3.1 and 3.2 discuss the case of failure-free elementary cells, and Section 3.3 presents the equivalent optimization under the occurrence of link failures. Finally, Section 3.4 discusses the extension of the optimization under an elementary cell into larger multihop topologies.

\subsection{Proposed Cross-Layer Optimization for an Elementary Cell without Link Failures}

To realize the optimal packet diversity within each elementary cell under no link failures, we introduced a new network-aware distortion reduction metric $\Delta_{v}^{r}$ as an extension of $\Delta_{v}$ of (2). Prior to the initial packet transmission, we have $\Delta_{v}^{r}=\Delta_{v}$. Each time the packet is successfully transmitted from the source node of the current cell (that is, an acknowledgment MSDU frame is received from an intermediate node of the cell), $\Delta_{v}^{r}$ is updated based on the remaining (expected) probability of error for the transmission of the packet in the remaining part of the elementary cell beyond the information horizon. Consider the topology in Fig. 2a. When packet $v$ is successfully transmitted over path $\mathbf{p}_{1}$, an acknowledgment MSDU frame is received from n1. Then, we can evaluate $\Delta_{v}^{r}$ as

$$
\Delta_{v}^{r}=\Delta_{v} \cdot \mathrm{E}\left\{e_{l_{1,2}}\left(L_{v}\right)\right\}
$$

The last equation indicates that, if we expect the packet to be dropped by link $l_{1,2}$, that is, $\mathrm{E}\left\{e_{l_{1,2}}\left(L_{v}\right)\right\}=1$, there is a remaining benefit (utility) in retransmitting the same packet over the alternate path $\left(\mathbf{p}_{2}\right)$, which is given by $\Delta_{v}^{r}=\Delta_{v}$. Conversely, if $\mathrm{E}\left\{e_{l_{1,2}}\left(L_{v}\right)\right\}=0$, there is no remaining benefit in retransmitting packet $v$. Therefore, $\Delta_{v}^{r}$ captures the expected distortion reduction achievable if a networkaware path diversity is chosen for packet $v$.

Our overall objective is to maximize the expected distortion reduction at the destination node of each elementary cell under the constraint of limited network resource and the deadline constraint of the video streaming application. Similar to our previous work [5], this is achieved by finding the "best path" as well as the crosslayer parameters for the link corresponding to that path:

$$
\begin{aligned}
& {\left[\begin{array}{llll}
v^{*} & \mathbf{p}_{i}^{*} & N_{\mathbf{p}_{i}}^{\max ^{*}} & m\left(l_{i, j}\right)
\end{array}\right]} \\
& =\arg \max _{\mathbf{p}_{i} \in \mathcal{P}, v \in \mathbf{v}_{\text {schedule }}(\mathrm{n} 0)}\left\{\left[1-e_{\mathbf{p}_{i}}\left(L_{v}\right)^{N_{\mathbf{p}_{i}}^{\max }}\right] \cdot \Delta_{v}^{r}\right\},
\end{aligned}
$$

where $\mathbf{v}_{\text {schedule }}(\mathrm{n} 0)$ is the set of nonexpired packets in the buffer of source node n0.

The expected end-to-end delay of the path should be smaller than the deadline of the packet; otherwise, the packet is dropped. This constraint can be expressed as

$$
\begin{aligned}
& \forall v \text {, and } \forall \mathbf{p}_{i} \in \mathcal{P}: N_{\mathbf{p}_{i}}^{\max } \leq \\
& \left\lfloor\frac{d_{v}^{\text {deadline }}-\mathrm{E}\left\{d_{\text {queue }}\left(l_{i, 2}\right)\right\}}{L_{v} \cdot\left(\left[g\left(l_{i, j}\right)\right]^{-1}+\left[\mathrm{E}\left\{g\left(l_{i, j}\right)\right\}\right]^{-1}\right)+2 \cdot T_{\text {overhead }}}-1\right.
\end{aligned}
$$

Notice that the bandwidth limitation of each link is only considered in the constraint of (11) and is not explicitly expressed in the optimization of (10) since each video packet is transmitted at the MAC layer until an acknowledgment is received or the delay deadline for the video packet is reached.

The algorithm in Fig. 4 can be deployed at the source node of each elementary cell in order to solve (10) under the constraint of (11). Note that, if multiple copies of the same packet come into the queue of an elementary cell source node from previous cells, we retain only one packet for transmission within the current cell. Finally, if the source node can utilize more than one cell, such as in the case in Fig. 3a, the algorithm in Fig. 4 is applied for all available outgoing links and, once a path has been chosen, the packet is considered within the corresponding elementary cell.

\subsection{Theoretical Analysis of the Failure-Free Case}

Under the optimization of (10), if the two paths have unequal error probabilities, then the path $\mathbf{p}_{i}$ with the lower 


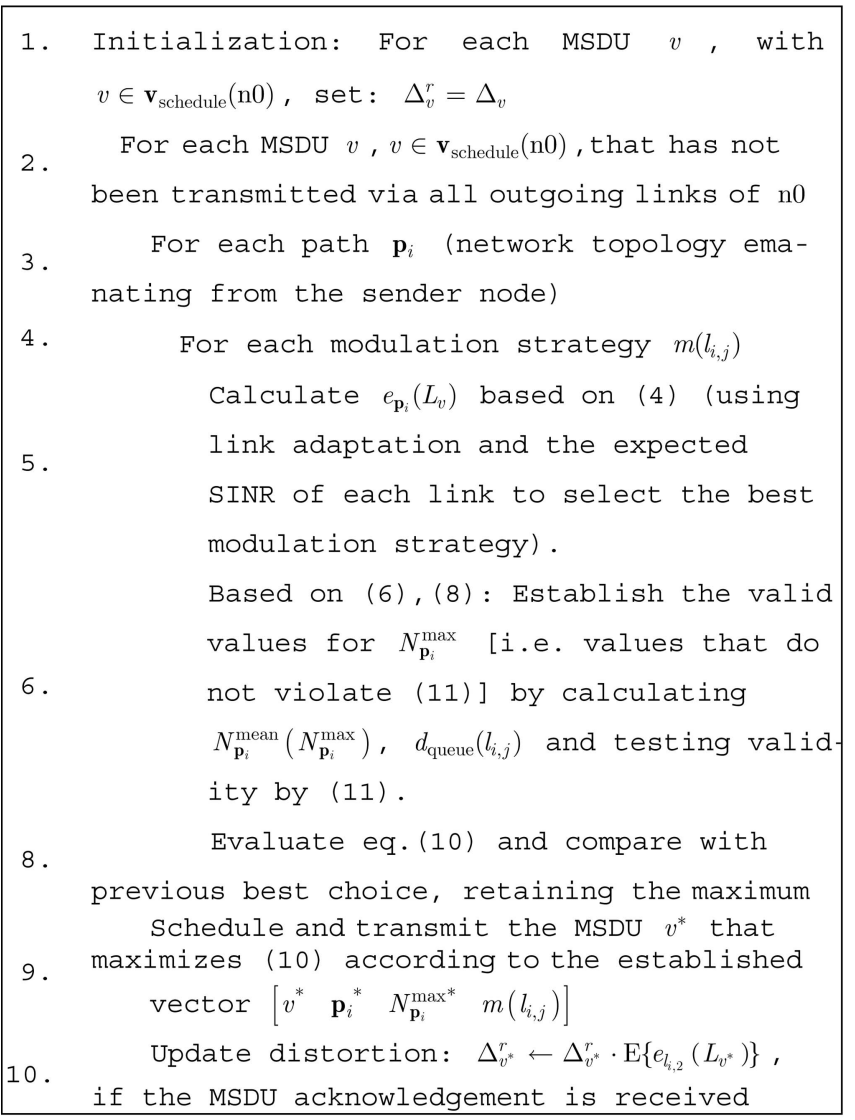

Fig. 4. Solution of cross-layer optimization of (10) for the sender node of an elementary cell. The utilized modulation strategy for link adaptation at Step 5 is derived based on previous modeling work for link adaptation strategies [19], [20].

error probability will be used to transmit packets, which will lead to a larger queuing delay for that path and, thereby, a smaller value for $N_{\mathbf{p}_{i}}^{\max }$ (from (11)). Thus, the term $\left[1-e_{\mathbf{p}_{i}}\left(L_{v}\right)^{N_{\mathbf{p}_{i}}^{\max }}\right]$ will decrease and, eventually, the alternate path $\mathbf{p}_{3-i}$ in the elementary cell will be chosen. Consequently, in the steady-state operation of each elementary cell, we may assume that ${ }^{4}$

$$
\left[e_{\mathbf{p}_{1}}\left(\overline{L_{v}}\right)\right]^{N_{\mathbf{p}_{1}}^{\max }}=\left[e_{\mathbf{p}_{2}}\left(\overline{L_{v}}\right)\right]^{N_{\mathbf{p}_{2}}^{\max }},
$$

where $\overline{L_{v}}$ is the nominal (average) packet size and

$$
\begin{aligned}
e_{\mathbf{p}_{i}} & =1-\left(1-e_{l_{i, 1}}\left(\overline{L_{v}}\right)\right)\left(1-\mathrm{E}\left\{e_{l_{i, 2}}\left(\overline{L_{v}}\right)\right\}\right) \\
& =1-\left(1-e\left(l_{i, 1}\right)\right)^{\overline{L_{v}}}\left(1-\mathrm{E}\left\{e\left(l_{i, 2}\right)\right\}\right)^{\overline{L_{v}}} \quad i=1,2 .
\end{aligned}
$$

Notice that $N_{\mathbf{p}_{i}}^{\max }\left(\overline{L_{v}}\right)$ can be determined based on (11) under the equality condition.

\subsubsection{Estimation of the Average Number of Successfully Transmitted Video Packets}

The queuing delay over path $\mathbf{p}_{i}$, that is, $d_{\text {queue }}\left(\mathbf{p}_{i}\right), i=1,2$, can be evaluated by modeling each path's intermediate nodes as an $M / G / 1$ queuing system. We assume that the

4. Equation (12) does not hold only under the trivial case when the capacity of one path is sufficient to transmit all the video packets (under the same deadline) with a smaller error probability than that of another path. In this case, no path diversity is beneficial. video packets arrive at the intermediate nodes following a Poisson process arrival rate $\lambda_{\text {total }}$, and each packet independently chooses path $\mathbf{p}_{i}$ at the sender node with probability $\operatorname{Pr}\left\{\mathbf{p}_{i}\right\}$. Thus, the packets arrive at each intermediate node $\mathrm{n} i(i=1,2)$ with average rate $\lambda_{i}=\lambda_{\text {total }} \cdot \operatorname{Pr}\left\{\mathbf{p}_{i}\right\}$.

By the Pollaczek-Khinchin (P-K) formula [22] and after some straightforward manipulations, the expected queuing delay $d_{\text {queue }}\left(\mathbf{p}_{i}\right)$ is given as

$$
d_{\text {queue }}\left(\mathbf{p}_{i}\right)=\frac{\lambda_{i} \cdot \mathrm{E}\left\{X_{i, \text { serv }}^{2}\right\}}{2\left(1-\lambda_{i} \cdot \mathrm{E}\left\{X_{i, \text { serv }}\right\}\right)},
$$

where

$$
\begin{aligned}
& \mathrm{E}\left\{X_{i, \text { serv }}\right\}= \\
& \left.\sum_{n=1}^{N_{\mathbf{p}_{i}}^{\max }}\left\{n \cdot \frac{\overline{L_{v}}}{\mathrm{E}\left\{g\left(l_{i, 2}\right)\right.}\right\} \cdot \mathrm{E}\left\{e_{l_{i, 2}}\left(\overline{L_{v}}\right)\right\}^{n-1} \cdot\left(1-\mathrm{E}\left\{e_{l_{i, 2}}\left(\overline{L_{v}}\right)\right\}\right)\right\} \\
& +\left(N_{\mathbf{p}_{i}}^{\max }+1\right) \frac{\overline{L_{v}}}{\mathrm{E}\left\{g\left(l_{i, 2}\right)\right\}} \cdot \mathrm{E}\left\{e_{l_{i, 2}}\left(\overline{L_{v}}\right)\right\}^{N_{\mathbf{p}_{i}}^{\max }}, \\
& \mathrm{E}\left\{X_{i, \text { serv }}^{2}\right\}= \\
& \sum_{n=1}^{N_{\mathbf{p}_{i}}^{\max }}\left\{\left(\frac{n \cdot \overline{L_{v}}}{\mathrm{E}\left\{g\left(l_{i, 2}\right)\right\}}\right)^{2} \cdot \mathrm{E}\left\{e_{l_{i, 2}}\left(\overline{L_{v}}\right)\right\}^{n-1} \cdot\left(1-\mathrm{E}\left\{e_{l_{i, 2}}\left(\overline{L_{v}}\right)\right\}\right)\right\} \\
& +\left(\left(N_{\mathbf{p}_{i}}^{\max }+1\right) \frac{\overline{L_{v}}}{\mathrm{E}\left\{g\left(l_{i, 2}\right)\right\}}\right)^{2} \cdot \mathrm{E}\left\{e_{l_{i, 2}}\left(\overline{L_{v}}\right)\right\}^{N_{\mathbf{p}_{i}}^{\max }}
\end{aligned}
$$

are the first and second moments of the service time $X_{i \text {,serv }}$ corresponding to the queue of path $\mathbf{p}_{i}$. With the knowledge of $d_{\text {queue }}\left(\mathbf{p}_{i}\right)$ from (14), we can establish $N_{\mathbf{p}_{i}}^{\text {mean }}\left(N_{\mathbf{p}_{i}}^{\max }\right)$ from (11) and (6). Then, the expected delay incurred by the bottleneck link of the path is given by

$$
d_{\text {bottleneck }}\left(\mathbf{p}_{i}\right)=N_{\mathbf{p}_{i}}^{\operatorname{mean}}\left(N_{\mathbf{p}_{i}}^{\max }\right) \cdot \overline{L_{v}} \cdot \max \left\{\frac{1}{g\left(l_{i, 1}\right)}, \frac{1}{\mathrm{E}\left\{g\left(l_{i, 2}\right)\right\}}\right\}
$$

based on the fact that each packet is transmitted on average $N_{\mathbf{p}_{i}}^{\text {mean }}\left(N_{\mathbf{p}_{i}}^{\max }\right)$ times at the MAC layer of each link, as calculated by the analysis of (3)-(6).

We can now establish the expected number of transmitted packets in the duration of one GOP (that is, under the transmission deadline) as

$$
S_{\mathrm{GOP}}=\left\lfloor d_{v}^{\text {deadline }} \cdot\left(\frac{1}{d_{\mathrm{bottleneck}}\left(\mathbf{p}_{1}\right)}+\frac{1}{d_{\text {bottleneck }}\left(\mathbf{p}_{2}\right)}\right)\right\rfloor .
$$

\subsubsection{Distortion Reduction under Failure-Free Transmission with and without Path Diversity}

Given that $S_{\mathrm{GOP}}$ packets are transmitted, based on the predetermined distortion prioritization scheme, the received distortion reduction when no link failure happens and without path diversity is given as

$$
\Delta_{\mathrm{GOP}}\{\text { fail-free, no-diversity }\}=\sum_{v=1}^{S_{\mathrm{GOP}}}\left[\alpha \cdot \rho^{v}\right]=\alpha \cdot \frac{\rho-\rho^{S_{\mathrm{GOP}}+1}}{1-\rho},
$$


where we utilized the proposed packet distortion reduction model of (2).

Under the use of path diversity, before attempting to retransmit packet $v$ over the alternate path, we assume to have transmitted $k_{\mathrm{dp}}$ subsequent packets, that is, packet $v+1$ to packet $v+k_{\mathrm{dp}}$, whose distortion reduction is greater than $\Delta_{v}^{r}$ calculated in Step 8 in Fig. 4. Obviously, $k_{\mathrm{dp}}$ depends on the distortion reductions $\left\{\Delta_{v+1}, \ldots, \Delta_{v+k_{\mathrm{dp}}}\right\}$ and $\Delta_{v}^{r}$. In the steady state, if packet $v$ is to be attempted over one path, it has a priori expected distortion reduction (that is, distortion reduction without knowing if an acknowledgment will be received or not)

$$
\Delta_{v}^{r}=\alpha \cdot \rho^{v} \cdot\left[e_{\mathbf{p}_{1}}\left(\overline{L_{v}}\right)\right]^{N_{\mathbf{p}_{1}}^{\max }}=\alpha \cdot \rho^{v} \cdot\left[e_{\mathbf{p}_{2}}\left(\overline{L_{v}}\right)\right]^{N_{\mathbf{p}_{2}}^{\max }}
$$

since both paths are balanced. We can compute an estimate of $k_{\mathrm{dp}}$ by equalizing $\Delta_{v}^{r}$ of packet $v$ expressed by (20) with the (initial) distortion reduction of packet $v+k_{\mathrm{dp}}$ :

$$
\alpha \cdot \rho^{v} \cdot\left[e_{\mathbf{p}_{1}}\left(\overline{L_{v}}\right)\right]^{N_{\mathbf{p}_{1}}^{\max }}=\alpha \cdot \rho^{v+k_{\mathrm{dp}}}, \quad i=\{1,2\},
$$

which gives

$$
k_{\mathrm{dp}}=\left\lfloor\log _{\rho}\left(\left[e_{\mathbf{p}_{1}}\left(\overline{L_{v}}\right)\right]^{N_{\mathbf{p}_{1}}^{\max }}\right)\right\rfloor, \quad i=1,2 .
$$

Under the steady-state assumption, the derived $k_{\mathrm{dp}}$ of (22) expresses the average number of packets between the original transmission of packet $v$ and its retransmission over the alternate path. Eventually, all initial packets of the current GOP will be retransmitted to the alternate path except for the last packets $\left\{S_{\mathrm{GOP}}-k_{\mathrm{dp}}, \ldots, S_{\mathrm{GOP}}\right\}$, which will expire before they can be replicated in the alternate path. In addition, in the steady-state case, each path is expected to successfully transmit $S_{\mathrm{GOP}} / 2$ packets in total. Hence, in the path diversity case with no link failures, the expected number of successfully received unique (that is, nonreplicated) video packets from both paths is

$$
S_{\mathrm{GOP}}^{\text {diff, fail-free }}=\left\lfloor\frac{S_{\mathrm{GOP}}+k_{\mathrm{dp}}}{2}\right\rfloor .
$$

Consequently, the received distortion reduction for the path diversity scheme can be calculated by

$$
\begin{aligned}
& \Delta_{\mathrm{GOP}}\{\text { fail-free, diversity }\}=\sum_{v=1}^{S_{\mathrm{GOP}}^{\text {diff fail-free }}}\left[\alpha \cdot \rho^{v}\right] \\
& =\alpha \cdot \frac{\rho-\rho^{S_{\mathrm{GOP}}^{\text {diff fail-free }}+1}}{1-\rho} .
\end{aligned}
$$

The comparison between the cases with no diversity and the case where path diversity is used can be performed by comparing $\Delta_{\mathrm{GOP}}\{$ fail-free, diversity $\}$ from (24) and $\Delta_{\mathrm{GOP}}\{$ fail-free, no-diversity $\}$ given in (19). The theoretical comparison reveals that path diversity will never increase the expected distortion reduction at the decoder since

$$
\begin{aligned}
& \Delta_{\mathrm{GOP}}\{\text { fail-free, diversity }\}> \\
& \Delta_{\mathrm{GOP}}\{\text { fail-free, no-diversity }\} \text { iff } k_{\mathrm{dp}}>S_{\mathrm{GOP}} .
\end{aligned}
$$

However, from the definition of $k_{\mathrm{dp}}, k_{\mathrm{dp}} \leq S_{\mathrm{GOP}}$. Consequently, path diversity should not be used under the crosslayer optimization in Fig. 4 when no failures occur in the multihop elementary cell. This conclusion is experimentally validated by our simulations and the corresponding analysis in Section 4. Section 3.3 examines the usefulness of path diversity under the occurrence of random link failures in the elementary cell topology.

\subsection{Retransmission and Path Diversity Optimization in the Case of Elementary Cells with Link Failures}

Under the occurrence of link failures beyond the horizon of the elementary cell topology, one cannot estimate the remaining distortion reduction for each packet in a reliable manner since this packet may be dropped in the intermediate node of the elementary cell topology. Hence, instead of using path diversity for a video packet based on the acknowledgment of the original packet transmission, we de facto retransmit a certain percentage of the most important video packets on both paths. Consequently, the algorithm in Fig. 4 is also applied for this case but with the modification that the original distortion reductions are used, that is, Step 10 in Fig. 4 is not applied. Moreover, the $\gamma \cdot 100$ percent most important packets $(0<\gamma \leq 1)$ in terms of distortion reduction are attempted on both available links in the elementary cell topology. The analysis in this section derives the dependency of $\gamma$ with the expected distortion reduction at the destination node.

For the theoretical study of this approach, the expected distortion reduction of the retransmission scheme in the case of link failures can be evaluated as

$$
\begin{aligned}
\Delta & \{\text { failure, diversity }\} \\
= & \Delta\{\text { fail-free, no-diversity }\} \cdot\left[\left(1-\mathrm{E}\left\{f\left(l_{1,2}\right)\right\}\right)\left(1-\mathrm{E}\left\{f\left(l_{2,2}\right)\right\}\right)\right. \\
& \left.+\sum_{i=1}^{2}\left[\left(1-\mathrm{E}\left\{f\left(l_{i, 2}\right)\right\}\right) \cdot \mathrm{E}\left\{f\left(l_{3-i, 2}\right)\right\} \cdot \frac{d_{v}^{\text {deadline }}}{d_{\text {bottleneck }}\left(\mathbf{p}_{i}\right) \cdot S_{\mathrm{GOP}}}\right]\right]
\end{aligned}
$$

where $\mathrm{E}\left\{f\left(l_{i, 2}\right)\right\}, i=\{1,2\}$, is the expected failure rate of link $l_{i, 2}, \Delta$ \{fail-free, no-diversity $\}$ is given by (19), and $d_{\text {bottleneck }}\left(\mathbf{p}_{i}\right)$ is given by (17). The last equation describes the expected distortion reduction under all possible combinations of failed paths and under the case of failure-free transmission.

The percentage of packets using path diversity should satisfy the following constraint:

$$
\gamma \leq\left[\frac{2}{S_{\mathrm{GOP}}} \min _{i=\{1,2\}}\left\{\frac{d_{v}^{\text {deadline }}}{d_{\text {bottleneck }}\left(\mathbf{p}_{i}\right)}\right\}\right] \cdot 100 \text { percent }
$$

because path diversity is inherently upper bounded by the bottleneck path. The last equation demonstrates that the maximum diversity can be achieved under equal congestion conditions on both paths, that is,

$$
d_{\text {bottleneck }}\left(\mathbf{p}_{1}\right)=d_{\text {bottleneck }}\left(\mathbf{p}_{2}\right),
$$

which leads to $\gamma \leq 100$ percent, that is, all video packets of each path could be retransmitted via the alternate path. The number of packets using path diversity is denoted by $S_{\mathrm{GOP}}^{\text {diversity }}$, and we have $S_{\mathrm{GOP}}^{\text {diversity }}=\gamma \cdot S_{\mathrm{GOP}}$. Similarly, the number of unique video packets transmitted via both paths in the case of failures is denoted by $S_{\mathrm{GOP}}^{\text {diff, fail }}$, and we have $S_{\mathrm{GOP}}^{\text {diff,fail }}=S_{\mathrm{GOP}}(1-0.5 \gamma)$. 
To simplify the notation, we define the expected number of packets transmitted through path $\mathbf{p}_{i}$ in the duration of one GOP as

$$
S_{\mathrm{GOP}}\left(\mathbf{p}_{i}\right)=\left\lfloor\frac{d_{v}^{\text {deadline }}}{d_{\text {bottleneck }}\left(\mathbf{p}_{i}\right)}\right\rfloor, i=1,2
$$

and we assume in the following analysis that $\gamma \cdot S_{\mathrm{GOP}}$ and $\frac{1}{2} \gamma \cdot S_{\mathrm{GOP}}$ are integers. Based on the utilized distortion model, the expected received distortion reduction of the diversity scheme can then be written as

$$
\begin{aligned}
& \Delta\{\text { failure, diversity }\} \\
& =\left(1-\mathrm{E}\left\{f\left(l_{1,2}\right)\right\}\right)\left(1-\mathrm{E}\left\{f\left(l_{2,2}\right)\right\}\right) \cdot \alpha \cdot \frac{\rho-\rho_{\mathrm{GOP}}^{\text {diff fail }}+1}{1-\rho} \\
& +\sum_{i=1}^{2}\left(1-\mathrm{E}\left\{f\left(l_{i, 2}\right)\right\}\right) \mathrm{E}\left\{f\left(l_{3-i, 2}\right)\right\} \\
& \cdot\left[\alpha \cdot \frac{\rho-\rho^{\frac{1}{2}} \int_{\mathrm{GOP}}^{\text {diverity }}+1}{1-\rho}+\frac{S_{\mathrm{GOP}}\left(\mathbf{p}_{i}\right)-\frac{1}{2} S_{\mathrm{GOP}}^{\text {diversity }}}{S_{\mathrm{GOP}}-S_{\mathrm{GOP}}^{\text {diversity }}}\right. \\
& \left.\cdot \alpha \cdot \rho^{\frac{1}{2} S_{\mathrm{GOP}}^{\text {diverity }}} \frac{\rho-\rho^{S_{\mathrm{GOP}}-S_{\mathrm{GOP}}^{\text {divesity }}+1}}{1-\rho}\right] \\
& =b_{1} \cdot \alpha \cdot \frac{\rho-\rho^{S_{\mathrm{GOP}}\left(1-\frac{1}{2} \gamma\right)+1}}{1-\rho} \\
& +b_{2} \cdot\left[\alpha \cdot \frac{\rho-\rho^{\frac{1}{2} \gamma} \cdot S_{\mathrm{GOP}}+1}{1-\rho}+\frac{S_{\mathrm{GOP}}\left(\mathbf{p}_{1}\right)-\frac{1}{2} \gamma \cdot S_{\mathrm{GOP}}}{S_{\mathrm{GOP}}(1-\gamma)}\right. \\
& \left.\cdot \alpha \cdot \rho^{\frac{1}{2}} \cdot S_{\mathrm{GOP}} \cdot \frac{\rho-\rho^{S_{\mathrm{GOP}}-\gamma \cdot S_{\mathrm{GOP}}+1}}{1-\rho}\right] \\
& +b_{3} \cdot\left[\alpha \cdot \frac{\rho-\rho^{\frac{1}{2}} \gamma \cdot S_{\mathrm{GOP}}+1}{1-\rho}+\frac{S_{\mathrm{GOP}}\left(\mathbf{p}_{2}\right)-\frac{1}{2} \gamma \cdot S_{\mathrm{GOP}}}{S_{\mathrm{GOP}}(1-\gamma)}\right. \\
& \left.\cdot \alpha \cdot \rho^{\frac{\gamma \cdot S_{\mathrm{GOP}}}{2}} \cdot \frac{\rho-\rho^{S_{\mathrm{GOP}}-\gamma \cdot S_{\mathrm{GOP}}+1}}{1-\rho}\right]
\end{aligned}
$$

with

$$
\left\{\begin{array}{l}
b_{1}=\left(1-\mathrm{E}\left\{f\left(l_{1,2}\right)\right\}\right)\left(1-\mathrm{E}\left\{f\left(l_{2,2}\right)\right\}\right) \\
b_{2}=\left(1-\mathrm{E}\left\{f\left(l_{1,2}\right)\right\}\right) \mathrm{E}\left\{f\left(l_{2,2}\right)\right\} \\
b_{3}=\left(1-\mathrm{E}\left\{f\left(l_{2,2}\right)\right\}\right) \mathrm{E}\left\{f\left(l_{1,2}\right)\right\}
\end{array}\right\} .
$$

After some straightforward manipulations, the distortion reduction expression of (30) is simplified to

$$
\begin{aligned}
& \Delta\{\text { failure, diversity }\}= \\
& c_{1}+\frac{c_{2}+c_{3} \cdot \gamma}{1-\gamma} \cdot \rho^{-\frac{1}{2} \gamma \cdot S_{\mathrm{GOP}}}+\frac{c_{4}+c_{5} \cdot \gamma}{1-\gamma} \cdot \rho^{\frac{1}{2} \gamma \cdot S_{\mathrm{GOP}}},
\end{aligned}
$$

where

$$
\left\{\begin{array}{l}
c_{1}=\left(b_{1}+b_{2}+b_{3}\right) \cdot \alpha \cdot \frac{\rho}{1-\rho} \\
c_{2}=-\left[b_{1}+b_{2} \cdot \frac{S_{\mathrm{GOP}}\left(\mathbf{p}_{1}\right)}{S_{\mathrm{GOP}}}+b_{3} \cdot \frac{S_{\mathrm{GOP}}\left(\mathbf{p}_{2}\right)}{S_{\mathrm{GOP}}}\right] \cdot \alpha \cdot \frac{\rho_{\mathrm{GOP}}+1}{1-\rho} \\
c_{3}=\left[b_{1}+\frac{b_{2}}{2}+\frac{b_{3}}{2}\right] \cdot \alpha \cdot \frac{\rho_{\mathrm{GOP}}+1}{1-\rho} \\
c_{4}=\left[b_{2} \cdot \frac{S_{\mathrm{GOP}}\left(\mathbf{p}_{1}\right)}{S_{\mathrm{GOP}}}+b_{3} \cdot \frac{S_{\mathrm{G}}\left(\mathbf{p}_{2}\right)}{S_{\mathrm{GOP}}}-\left(b_{2}+b_{3}\right)\right] \cdot \alpha \cdot \frac{\rho}{1-\rho} \\
c_{5}=\frac{b_{2}+b_{3}}{2} \cdot \alpha \cdot \frac{\rho}{1-\rho}
\end{array}\right\} .
$$

The expected distortion reduction under path failures given by (32) is a function of the packet diversity percentage $\gamma$. Hence, given the network parameters, we can analytically determine $\gamma$ to obtain the maximum video quality at the decoding node of the elementary cell. For example, when $\mathbf{p}_{1}$ and $\mathbf{p}_{2}$ have the same delay bottleneck, that is, $d_{\text {bottleneck }}\left(\mathbf{p}_{1}\right)=d_{\text {bottleneck }}\left(\mathbf{p}_{2}\right)$, we have $S_{\mathrm{GOP}}\left(\mathbf{p}_{1}\right)=$ $S_{\mathrm{GOP}}\left(\mathbf{p}_{2}\right)=\left\lfloor\frac{1}{2} S_{\mathrm{GOP}}\right\rfloor$ from (28). Then, (32) is simplified to

$$
\Delta\{\text { failure, diversity }\}=c_{1}+c_{2} \cdot \rho^{-\frac{1}{2} \gamma \cdot S_{\mathrm{GOP}}}+c_{4} \cdot \rho^{\frac{1}{2} \gamma \cdot S_{\mathrm{GOP}}} .
$$

By setting the first derivative of $\Delta$ \{failure, diversity $\}$ to zero, we obtain the (optimal) percentage of video packets using path diversity that is expected to maximize the distortion reduction under path failures:

$$
\gamma^{*}=\frac{1}{S_{\mathrm{GOP}}} \log _{\rho}\left(\frac{c_{2}}{c_{4}}\right)=\frac{1}{S_{\mathrm{GOP}}} \log _{\rho}\left(\frac{2 b_{1}+b_{2}+b_{3}}{b_{2}+b_{3}} \cdot \rho^{S_{\mathrm{GOP}}}\right) .
$$

Notice that the last expression gives the optimal packet diversity percentage as a function of the distortion reduction model $(\rho)$ and the constants of (31), which are expressing the expected failure conditions. However, the optimal value $\gamma^{*}$ is invariant to the scaling parameter $\alpha$ of the distortion model since all the video packets are equally influenced by it.

\subsection{Extensions to Large Topologies}

Large topologies consisting of 7-20 nodes can be decomposed into overlapping elementary cells, as shown in the example in Fig. 3. For each elementary cell, the sender node faces the situation discussed previously for the basic topology, that is, it has accurate information for its outgoing links and estimated information for the remainder of the elementary cell. We can categorize elementary cells into failure-free cells and cells with link failures. Each case follows the analysis presented previously in this section.

Although analytically tractable, video streaming following the elementary cell approach may lead to locally optimal solutions that do not correspond to the globally optimal video streaming approach that considers the network parameters for the entire multihop topology. However, one will need to cope with the large uncertainty in the parameter estimation when the entire multihop topology is considered due to frequent variations in the link conditions in wireless multihop networks. Although this may be partially alleviated if the sender node receives frequent updates for each link's condition, such an approach may incur significant bandwidth overheads and delays. The elementary cell approach provides the intermediate solution, that is, high performance with reasonable feedback requirements about the network status. However, since the performance gap from the end-to-end optimized solution may become significant when large topologies are considered in the following section, we quantify the performance of the elementary cell approach against the upper bound "oracle" approach that assumes instantaneous feedback about the link conditions of the entire network (including precise knowledge of link failures) and applies the cross-layer optimization of Fig. 4 for the entire topology. 


\section{Simulation Results}

In our experiments, we used a fully scalable codec [23] and the produced bitstream for each video sequence was extracted at an average bit rate of 2 megabits per second (Mbps) for CIF resolution sequences at 30 frames per second. Based on the rate-distortion hint track specification [15], each video bitstream was packetized into MSDUs of data payload no larger than 1,000 bytes. Each GOP was set to 16 video frames. The end-to-end delay for the MSDUs of each GOP was set to $0.533 \mathrm{sec}$, which corresponds to the replay duration of one GOP (at $30 \mathrm{~Hz}$ ) and is also an acceptable latency for the majority of real-time video streaming applications.

For the experimental study of the elementary cell topology, we simulated the case of the multihop network shown in Fig. 2a. Moreover, we also considered the larger multihop network in Fig. 3 in order to validate our study of elementary cells within a larger multihop topology. In all cases, we assume that the central coordinator [11] or the distributed overlay infrastructure [12], [13] provides predetermined transmission intervals for each link corresponding to the indicated application-layer bandwidth (in Kbps) for each experiment. Under the knowledge of the SINR of each link and the utilized modulation strategy, this leads to known guaranteed bandwidth and BER per link. However, since each link's SINR may vary unpredictably, in our theoretical analysis, we always use the average SINR of each link that leads to the expected packet loss rate and the average guaranteed bandwidth under the predetermined transmission interval for the video flow.

In order to make a realistic evaluation of the dynamics of multihop wireless topologies, we performed a parameterized simulation that takes into account the different options and settings for the various layers, such as varying SINR at the PHY layer, transmission overheads at the MAC layer due to MSDU acknowledgments, and polling, as well as queuing and propagation delays in the various links of the multihop network. In order to incorporate the effect of noise and interference, we performed 50 simulations per topology/video sequence/chosen transmission setting using random values for the SINR of each link, chosen between 17 and $23 \mathrm{~dB}$. This range was selected such that the 802.11a modulation strategies at the PHY provide reasonable packet loss rates with enough PHY layer throughput to support video applications (see the modulation curves in [19] and [20] for more details on packet loss rates and throughput versus SINR). In Section 4.1, we first validate the utilized distortion reduction model of (2) using typical video sequences. Then, Sections 4.2 and 4.3 examine the elementary cell topology and validate the proposed theoretical analysis for the failure-free case and under the case of link failures. Finally, Section 4.4 presents results for video streaming under a larger multihop topology when the optimization is performed within elementary cells. A comparison is made with the globally optimized streaming solution, which assumes exact knowledge of the network conditions.

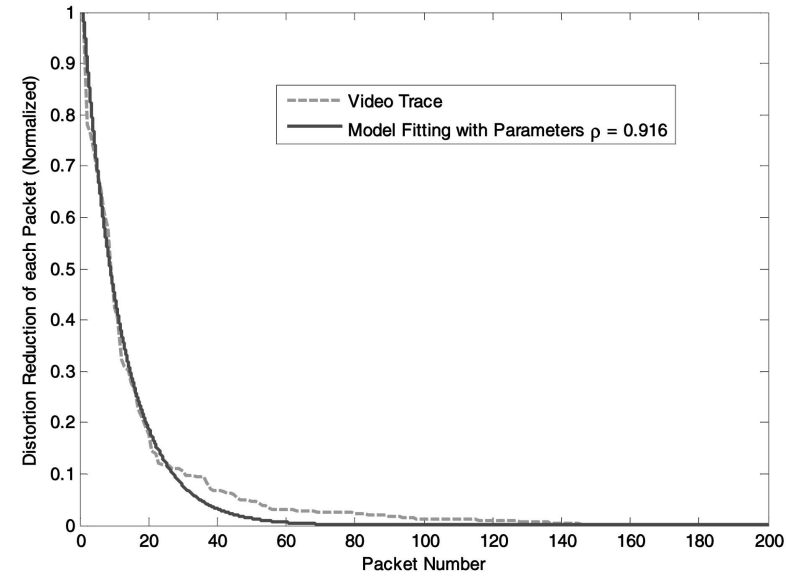

(a)

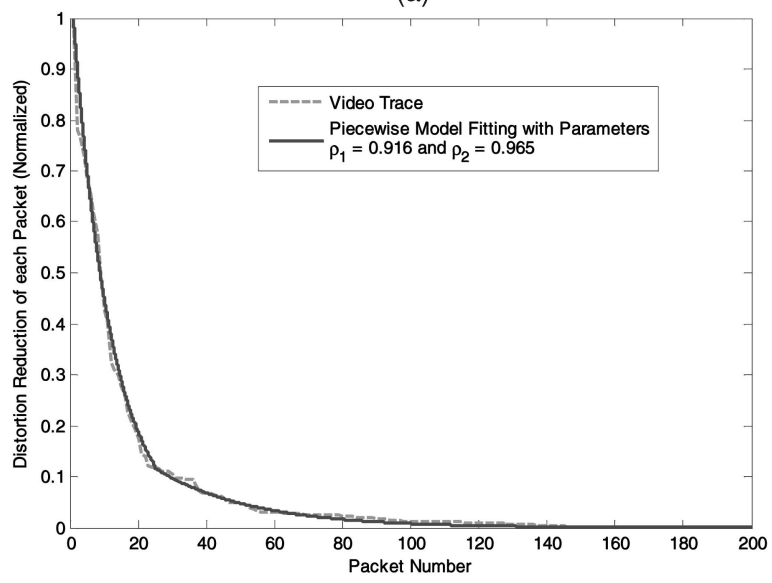

(b)

Fig. 5. Minimum mean square error (MSE) fitting of the model and real video trace for (a) an entire GOP and (b) two groups of packets (piecewise approximation). The sequence "Foreman" was used, and the decoding bit rate was 2 Mbps.

\subsection{Validation of the Video Packet Distortion Reduction Model}

Fig. 5a shows a typical comparison between the experimental distortion reduction of the packets in one GOP (computed by the video decoder for a GOP of sequence "Foreman") and the distortion reduction given by the proposed model. Similar results have been obtained for all GOPs of all the remaining video sequences of our experiments and under a variety of decoding bit rates. Under the proper selection of the model parameters for each GOP (that is, $a$ and $\rho$ of (2)), we always obtain a sufficient approximation of the distortion reduction for each packet. In addition, a piecewise distortion model can provide even higher accuracy, as shown in the example in Fig. $5 \mathrm{~b}$. In this case, the packets of each GOP are divided into $I_{G}$ groups, and each group is approximated by the proposed model:

$$
\Delta_{G O P}=\sum_{i=1}^{I_{G}} \alpha_{i} \cdot \rho_{i}^{v-m_{i}} \quad \rho_{i} \in(0,1), v=1,2,3, \ldots,
$$

where $\alpha_{i}$ and $\rho_{i}$ are the model parameters for the $i$ th group of video packets and $m_{i}$ is the packet number of the first packet in the $i$ th group, with $m_{1}=1$. In the particular 
TABLE 1

Comparison between Simulation Average and Theoretically Estimated Results for Failure-Free Low and High-Bandwidth Balanced Elementary Cells

\begin{tabular}{|c|c|c|c|c|}
\hline \multicolumn{5}{|c|}{$\begin{array}{l}\text { Common Parameter settings: } \overline{L_{v}}=750 \cdot 8 \text { bits } \\
\quad e_{l_{i, 1}}\left(\overline{L_{v}}\right)=0.1 \quad \mathrm{E}\left\{e_{l_{i, 2}}\left(\overline{L_{v}}\right)\right\}=0.2 \quad i=1,2\end{array}$} \\
\hline Low-bandwidth & & mulatio & bps & $\pi 1$ \\
\hline $\begin{array}{l}\text { Low-bandwidth } \\
\text { Scenario }\end{array}$ & Min & Mean & Max & Theoretical \\
\hline$S_{\mathrm{GOP}}$ & 17 & 26 & 34 & 28 \\
\hline $\begin{array}{r}\Delta_{\mathrm{GOP}}\{\text { fail-free }, \\
\text { no-diversity }\}\end{array}$ & 0.765 & 0.872 & 0.934 & 0.901 \\
\hline High-bandwidth & & mulatio & $\mathrm{n}$ & \\
\hline $\begin{array}{l}\text { High-bandwidth } \\
\text { Scenario }\end{array}$ & Min & Mean & Max & Theoretical \\
\hline$S_{\mathrm{GOP}}$ & 131 & 166 & 199 & 170 \\
\hline $\begin{array}{r}\Delta_{\mathrm{GOP}}\{\text { fail-free } \\
\text { no-diversity }\}\end{array}$ & 0.981 & 0.981 & 0.981 & 0.981 \\
\hline
\end{tabular}

We indicate the average received number of packets and the normalized distortion reduction measured from the simulation and estimated from the proposed formulation.

example in Fig. $5 \mathrm{~b}$ where $m_{1}=1$ and $m_{2}=26$, the distortion reduction of packets $v=\{1, \ldots, 25\}$ is approximated by $\alpha_{1}=1.0$ and $\rho_{1}=0.916$, and the distortion reduction of packets $v=\{26, \ldots, 200\}$ is approximated by $\alpha_{2}=0.119$ and $\rho_{2}=0.965$ using least squares fitting. Overall, we used $I_{G}=2$ for all video sequences, but the values for $\alpha_{\{1,2\}}$ and $\rho_{\{1,2\}}$ are sequence-dependent and are defined based on least squares fitting with the experimental distortion estimates of the scalable codec, as produced during the bitstream extraction phase [23].

\subsection{Experimental Results and Model Validation for Failure-Free Elementary Cells}

In our first round of experiments, we separated our analysis into two cases corresponding to low and high average bandwidth for the video transmission. Thereafter, we examine a balanced and an unbalanced topology (in terms of estimated available bandwidth for each path).

Representative results for the first case are shown in Table 1. The average guaranteed bandwidth and average packet loss rate of each link displayed in the table were calculated based on the average SINR and the corresponding modulation scheme for the average interference case, although different modulations were utilized during the simulation of the MSDU transmission using link adaptation
TABLE 2

Comparison between Simulation Average and Theoretically Estimated Results for Unbalanced Elementary Cells

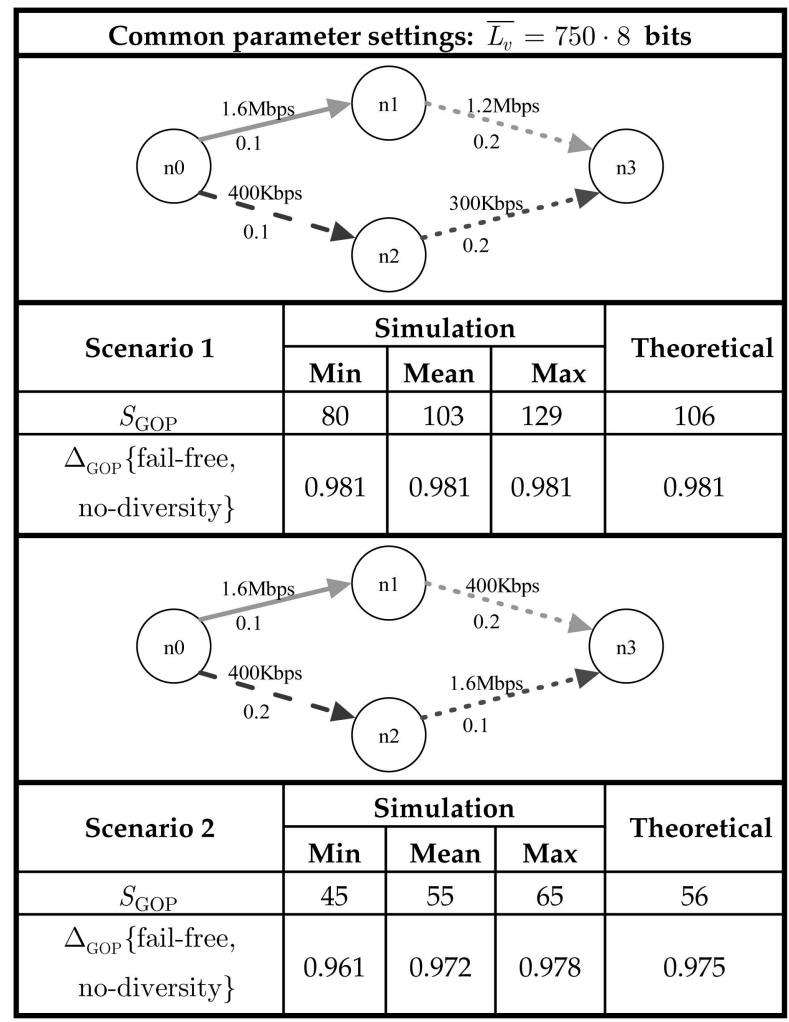

We indicate the average received number of packets and the distortion reduction measured from the simulation and estimated from the proposed formulation.

at the PHY layer [19], [20]. Although the channel conditions vary depending on interference, we find that, even with unbalanced packet errors for the two paths, the received packets are evenly distributed over the two paths under the scenarios in Table 1 . This is because we incorporate the queuing delay in the evaluation of $N_{\mathbf{p}_{i}}^{\max }$ in (11), and this affects the expected packet loss of the optimization function of (10). Hence, if too many packets are transmitted over one path, the queuing delay of this path will grow, which will result in a lower maximum packet retransmission limit and, thus, a larger path error rate. Then, the sender node will switch to the alternate path.

The second set of our experiments is outlined by the representative results in Table 2. In scenario 1, we found that on average there are 82.15 packets transmitted over path 1 and 21.55 packets transmitted over path 2; thus, the load is distributed over the two paths according to the path bandwidth ratio. Similar results are observed for the scenario with larger error rates, different bandwidths, and packet size variation. The results in Tables 1 and 2 indicate that the proposed theoretical estimation captures the average simulated behavior accurately.

Concerning the case of path diversity in the failure-free elementary cell topology, our simulation validated that it is not a beneficial approach. In fact, we observed that the proposed algorithm in Fig. 4 very rarely (if ever) selects to retransmit a video packet on the alternate path, which 


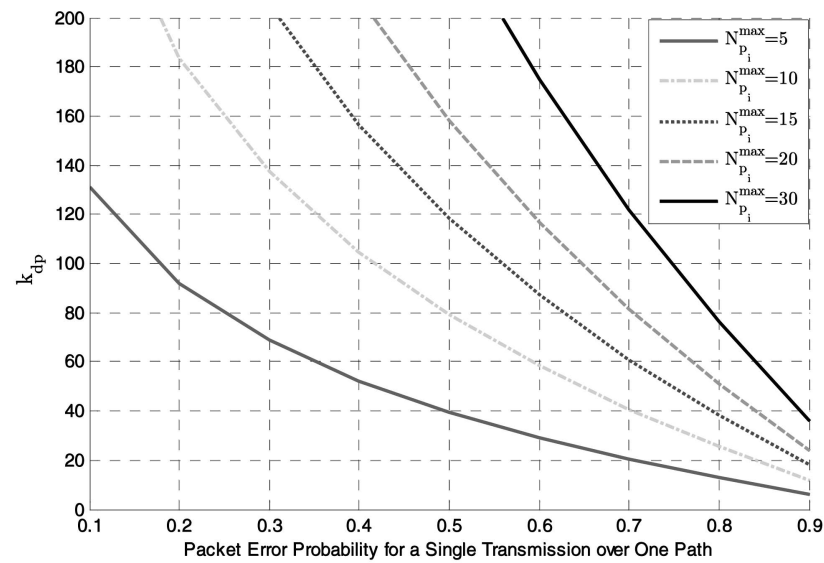

Fig. 6. Relationship of $k_{\mathrm{dp}}$ (the average number of packets between the original transmission of packet $v$ and its retransmission over the alternate path) with the retransmission limit of each path $\left(N_{\mathrm{p}_{i}}^{\max }\right)$ and the path error probability $\left(e_{\mathbf{p}_{i}}\left(L_{v}\right)\right)$. The plots are generated based on (22), which assumes a balanced elementary cell topology under a steady-state operation, and we set $\rho=0.916$, which agrees with experimentally measured distortion reduction in typical video sequences.

agrees with the derived theoretical outcome of (25). In order to elaborate further on why the proposed algorithm does not use the option of path diversity, Fig. 6 examines the relationship of the theoretically predicted value of $k_{\mathrm{dp}}$ from (22) with the path error probability for a single packet transmission $\left(e_{p_{i}}\left(L_{v}\right)\right)$ and under various retransmission limits $N_{\mathbf{p}_{i}}^{\max }, i=\{1,2\}$. The plots clearly demonstrate that, even for very high path error probability and for a large variety of retransmission limits (which correspond to relaxed or strict delay deadlines-since $N_{\mathbf{p}_{i}}^{\max }$ is defined based on (11)), the theoretical value of $k_{\mathrm{dp}}$ is larger than the retransmission limit $N_{\mathbf{p}_{i}}^{\max }$ for each path $\mathbf{p}_{i}$. These results indicate that, in failure-free multihop topologies and under a persistent MAC retransmission framework that attempts to transmit the most important MSDUs (in terms of distortion reduction) until the delay deadline is reached, path diversity does not provide an improved distortion performance, that is, it does not lead to higher distortion reduction at the decoder.

\subsection{Experimental Results and Model Validation in Elementary Cells Exhibiting Path Failures}

We consider again the low-bandwidth elementary cell indicated in the low-bandwidth case in Table 1. All undetected failures occur in the dotted links and they are handled by using path diversity as explained in Section 3.3. Although failures may also occur in the link connected to the source node $\mathrm{n} 0$, they are easily detected by the source node and, then, this case essentially becomes a single-path transmission problem. Hence, we focus our experimental study on failures for the "unknown" part of the multihop elementary cell, that is, beyond the first link of each path.

Fig. 7 shows the theoretically expected distortion reduction at the destination (decoder) node as a function of $\mathrm{E}\left\{f\left(l_{1,2}\right)\right\}$ (which is equal to $\mathrm{E}\left\{f\left(l_{2,2}\right)\right\}$ for this analysis) and the percentage of diversity. Clearly, when $\mathrm{E}\left\{f\left(l_{1,2}\right)\right\}=0$, this case rolls back to the previous situation, and using path diversity will always lead to lower distortion reduction

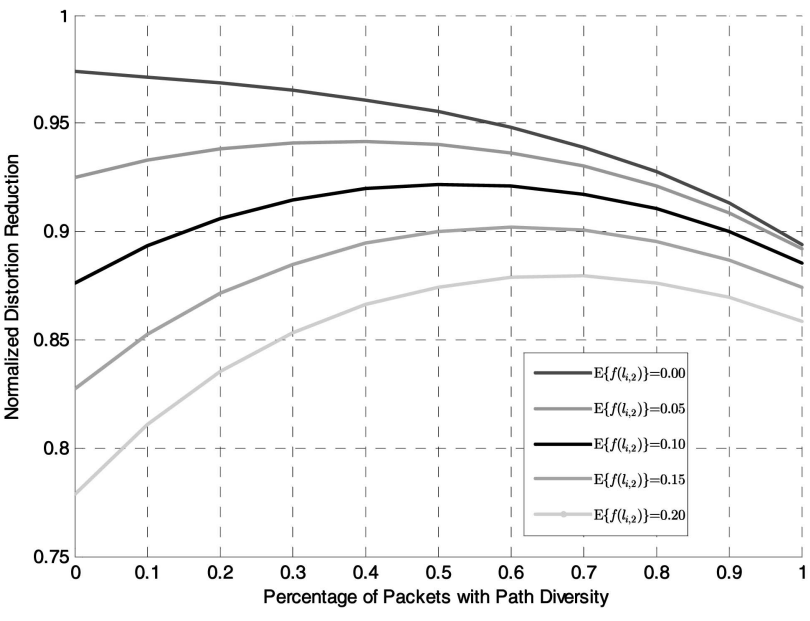

Fig. 7. Analytically evaluated distortion reduction for different link-failure probabilities as a function of the percentage of GOP packets using path diversity for the low-bandwidth elementary cell topology.

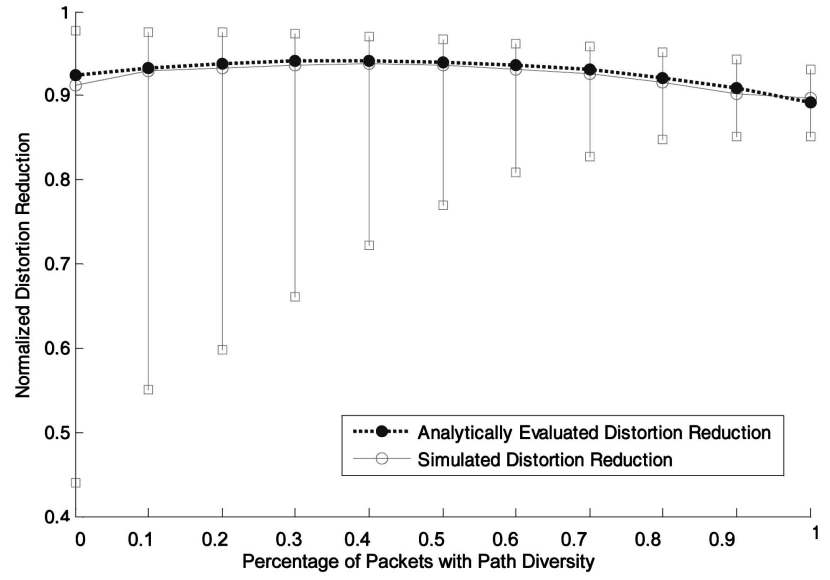

Fig. 8. Comparison of simulation and theoretical results for $\mathrm{E}\left\{f\left(l_{1,2}\right)\right\}=$ $\mathrm{E}\left\{f\left(l_{2,2}\right)\right\}=0.05$ in the low-bandwidth elementary topology.

compared with the no-path-diversity scheme (and the expected distortion reduction is monotonically decreasing as the percentage of packets using path diversity increases). However, it appears that, even for modest values of $\mathrm{E}\left\{f\left(l_{1,2}\right)\right\}$, packet diversity can achieve higher distortion reduction if the percentage of packets using diversity is chosen carefully. The theoretical curves in Fig. 7 clearly demonstrate that there exists a maximum value for the percentage of packets using path diversity, which was expressed in the conclusion of our analysis by (35). In addition, when path diversity is selected beyond the optimum point, although the average distortion reduction will decrease, the variance of the decoded video quality will also decrease, since fewer video packets are sacrificed during the occurrence of a link failure.

The detailed simulation results and the corresponding theoretical prediction for a variety of failure probabilities are presented in Figs. 8 and 9. The analytical model matches well with the simulation results. The bars in each figure illustrate the range of the distortion reduction observed in our simulations with each choice for path diversity percentage. Clearly, the variance of the distortion reduction 


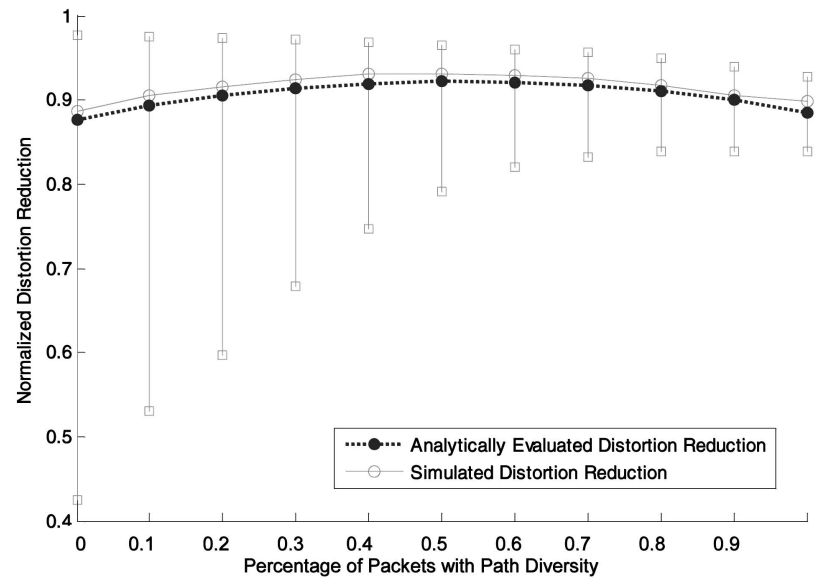

Fig. 9. Comparison of simulation and theoretical results for $\mathrm{E}\left\{f\left(l_{1,2}\right)\right\}=$ $\mathrm{E}\left\{f\left(l_{2,2}\right)\right\}=0.10$ in the low-bandwidth elementary topology.

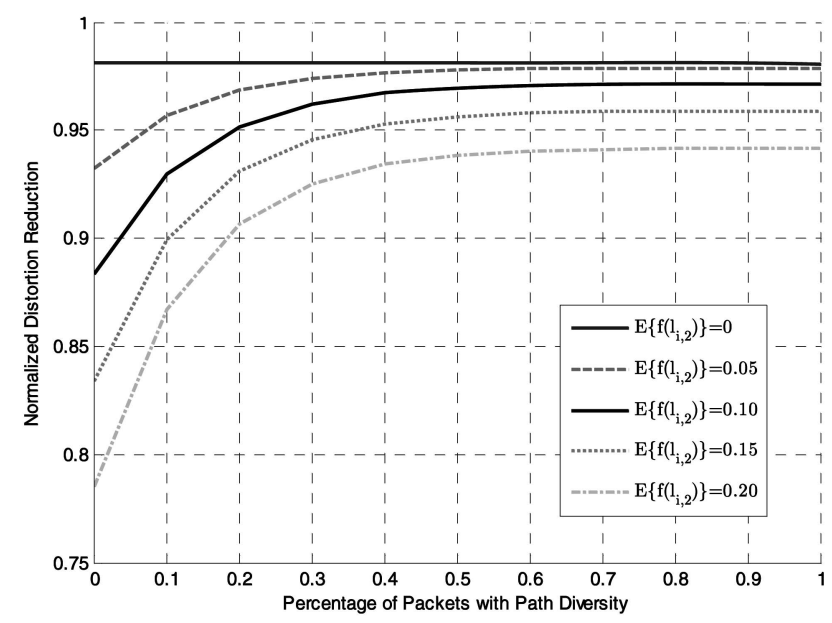

Fig. 10. Analytically evaluated distortion reduction for different linkfailure probabilities as a function of the percentage of GOP packets using path diversity for the high-bandwidth elementary cell topology.

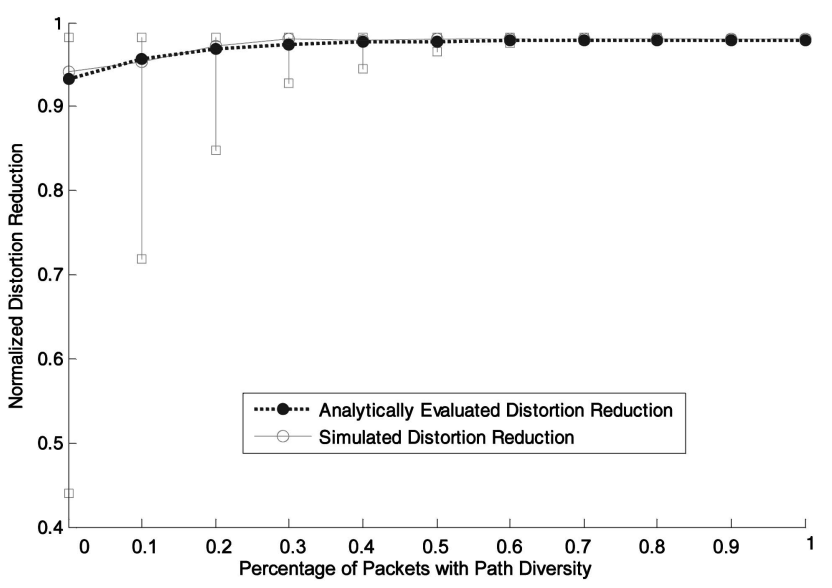

Fig. 11. Comparison of simulation and theoretical results for $\mathrm{E}\left\{f\left(l_{1,2}\right)\right\}=$ $\mathrm{E}\left\{f\left(l_{2,2}\right)\right\}=0.05$ in the high-bandwidth elementary topology.

at the decoder is reduced by increased percentages of packets using path diversity.

Concerning the high-bandwidth case in Table 1, the behavior under path failures is somewhat different than in the case of low bandwidth. In the former case, there is sufficient bandwidth to transmit the most important

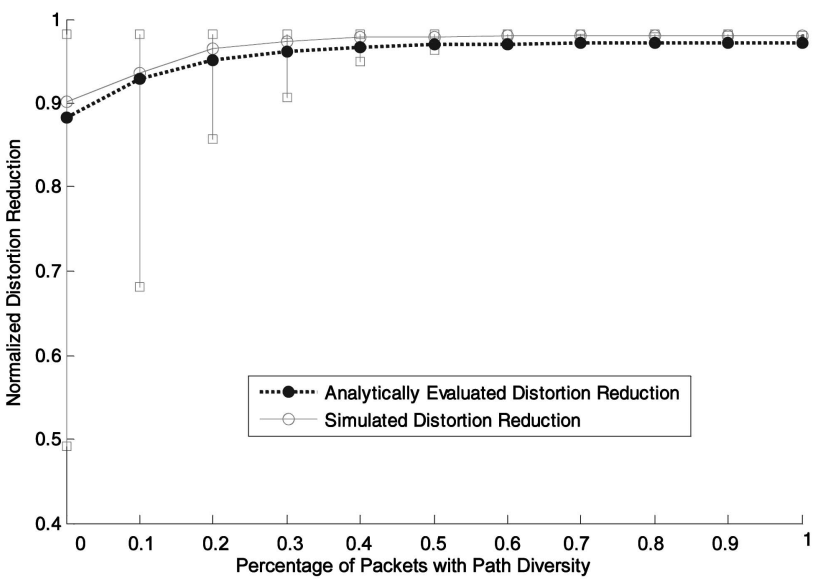

Fig. 12. Comparison of simulation and theoretical results for $\mathrm{E}\left\{f\left(l_{1,2}\right)\right\}=$ $\mathrm{E}\left\{f\left(l_{2,2}\right)\right\}=0.10$ in the high-bandwidth elementary topology.

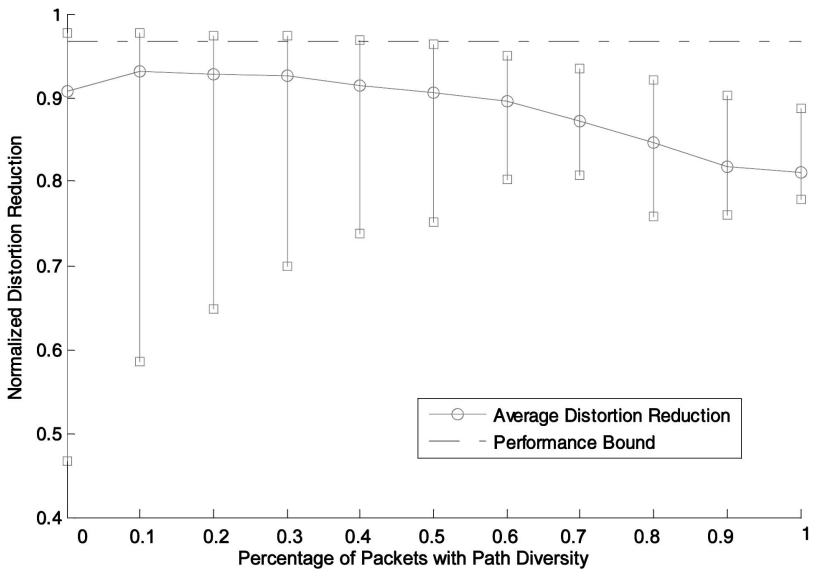

Fig. 13. Simulation results with the multihop topology in Fig. 3 under the occurrence of link failures.

packets of each GOP on both paths. Consequently, even when a large number of video packets use path diversity, there is no considerable decrease in average distortion reduction, as shown in the theoretical comparisons in Figs. 10, 11, and 12. Simulation results verify this fact and we find that, as the percentage of diversity becomes large, the received distortion reduction becomes very stable and the variance is negligible in these cases.

\subsection{Experimental Results for Larger Multihop Topologies}

We consider the entire multihop topology in Fig. 3 and simulate the case where all links operate with 5 percent failure probability for the duration of the video streaming session. For the reported experiments, all failures are assumed to be short-lived, that is, lasting approximately for an entire GOP (16 frames at replay rate of $30 \mathrm{~Hz}$, that is, $0.5 \mathrm{sec})$. The distortion reduction as a function of the percentage of packets utilizing path diversity is given in Fig. 13. The top horizontal line indicates the performance bound, which corresponds to the scheduling and crosslayer optimization that considers the end-to-end network parameters and assumes instantaneous feedback concerning link failures. This corresponds to the "end-to-end" optimization case of our recent work [5] with the additional 
TABLE 3

Simulation Results for Two Video Sequences under Various Failure Probabilities and Varying Percentage of Packets Utilizing Path Diversity

\begin{tabular}{|c|c|c|c|c|c|c|}
\hline \multirow{3}{*}{$\begin{array}{c}\text { Sequence "Fore- } \\
\text { man" }\end{array}$} & \multicolumn{3}{|c|}{ Failure Probability } & \multicolumn{3}{|c|}{ Failure Probability } \\
\hline & 0.1 & 0.05 & 0.03 & 0.1 & 0.05 & 0.03 \\
\hline & \multicolumn{3}{|c|}{ PSNR (dB) } & \multicolumn{3}{|c|}{$\begin{array}{c}\text { Lost Video Frames } \\
\text { (percentage) }\end{array}$} \\
\hline Performance Bound & 34.57 & 34.91 & 35.40 & $0 \%$ & $0 \%$ & $0 \%$ \\
\hline \multicolumn{7}{|l|}{ Diversity Percentage } \\
\hline 0 & 25.76 & 30.19 & 33.52 & $38 \%$ & $18.30 \%$ & $5.88 \%$ \\
\hline 0.3 & 33.60 & 34.37 & 34.80 & $0 \%$ & $0 \%$ & $0 \%$ \\
\hline 0.6 & 33.95 & 34.27 & 34.62 & $0 \%$ & $0 \%$ & $0 \%$ \\
\hline 1 & 33.82 & 33.82 & 33.83 & $0 \%$ & $0 \%$ & $0 \%$ \\
\hline \multirow{3}{*}{$\begin{array}{c}\text { Sequence } \\
\text { "Coastguard" }\end{array}$} & \multicolumn{3}{|c|}{ Failure Probability } & \multicolumn{3}{|c|}{ Failure Probability } \\
\hline & 0.1 & 0.05 & 0.03 & 0.1 & 0.05 & 0.03 \\
\hline & \multicolumn{3}{|c|}{ PSNR (dB) } & \multicolumn{3}{|c|}{$\begin{array}{c}\text { Lost Video Frames } \\
\text { (percentage) }\end{array}$} \\
\hline Performance Bound & 32.55 & 32.78 & 33.05 & $0 \%$ & $0 \%$ & $0 \%$ \\
\hline \multicolumn{7}{|l|}{ Diversity Percentage } \\
\hline 0 & 23.31 & 28.40 & 31.28 & $43 \%$ & $20.07 \%$ & $5.54 \%$ \\
\hline 0.3 & 30.59 & 31.59 & 32.19 & $8.30 \%$ & $4.84 \%$ & $0 \%$ \\
\hline 0.6 & 31.08 & 31.31 & 31.59 & $0 \%$ & $0 \%$ & $0 \%$ \\
\hline 1 & 31.32 & 31.31 & 31.33 & $0 \%$ & $0 \%$ & $0 \%$ \\
\hline
\end{tabular}

The bandwidth allocation per link is indicated in the top part. The corresponding packet loss rate of each link (for the failure-free intervals) was varying between 0.08 and 0.12 , derived based on the average packet size and the BER using (3).

knowledge of link-failure occurrences, which is in general not easily feasible due to the feedback requirements. Similar results have also been obtained when the failure probability is uniformly distributed within the range of $[0,10]$ percent, as well as when the link failures last longer.

Overall, the results indicate that our localized optimization mechanism within overlapping elementary cells consists of a good approximation of the globally optimal solution that assumes exact knowledge of the network conditions and link failures for the entire network. In addition, in this case, the main benefit of using path diversity appears to be the reduction of the expected variance of the distortion reduction at the destination node.

We validated these results by measuring the peak signalto-noise ratio (PSNR) for two typical Common Intermediate Format (CIF) video sequences under the simulation conditions in Table 3, as well as the average percentage of lost video frames. Since the quality of a lost video frame depends on the error concealment strategy applied at the decoder side, the percentage of lost video frames was quantified based on the number of video frames with PSNR lower than $26 \mathrm{~dB}$, which indicates that the video frame appears with severe distortion. The results indicate that path diversity is beneficial when link failures are frequent. Moreover, a higher percentage of video packets utilizing path diversity ensures that fewer video frames are severely distorted.

\section{Conclusions}

Wireless multihop networks may emerge as important infrastructures for video streaming applications. In this paper, we investigated the performance of such streaming applications under a cross-layer optimization framework that attempts to cope with rapidly varying network conditions and even complete link failures. Our approach splits the video streaming problem into independent transmission problems within overlapping "elementary cells" in the multihop network. Each cell represents an elementary multihop topology that enables cross-layer optimization with path diversity.

By utilizing state-of-the-art video coding with predetermined distortion reduction prioritization for each video packet, we modeled and analyzed the expected performance of the proposed framework under a variety of conditions. Our theoretical derivations demonstrate that, under the proposed cross-layer optimization framework, path diversity is not beneficial when link failures are not expected in the wireless multihop infrastructure. On the other hand, path diversity is a useful approach for lowbandwidth transmission over multihop networks if the percentage of video packets utilizing diversity is chosen carefully. Finally, for larger multihop topologies, the main benefit of utilizing path diversity appears to be the stabilization of the decoded video quality. In this case, the accurate choice of the percentage of video packets to use path diversity is not so critical. Our conclusions are supported by simulation studies with a variety of video sequences and a variety of network configurations. The simulation results additionally indicated that the proposed approach of using elementary cells performs close to the upper bound that considers the entire network topology during the cross-layer optimization and assumes instantaneous feedback about the link conditions.

\section{ACKNOWLEDGMENTS}

The authors would like to acknowledge the support from US National Science Foundation Grants CCF 0541867 and CNS 0509522. At the time of this work, Y. Andreopoulos was with the Department of Electrical Engineering, University of California, Los Angeles.

\section{References}

[1] E. Setton, T. Yoo, X. Zhu, A. Goldsmith, and B. Girod, "CrossLayer Design of Ad Hoc Networks for Real-Time Video Streaming," IEEE Wireless Comm. Magazine, vol. 12, no. 4, pp. 5965, Aug. 2005.

[2] N. Gogate, D.-M. Chung, S.S. Panwar, and Y. Wang, "Supporting Image and Video Applications in a Multihop Radio Environment Using Path Diversity and Multiple Description Coding," IEEE Trans. Circuits and Systems for Video Technology, vol. 12, no. 9, pp. 777-793, Sept. 2002. 
[3] I.F. Akyildiz, X. Wang, and W. Wang, "Wireless Mesh Networks: A Survey," Computer Networks J., vol. 47, no. 4, pp. 445-487, Mar. 2005.

[4] IEEE 802.11e/D5.0, Draft Supplement to Part 11: Wireless Medium Access Control (MAC) and Physical Layer (PHY) Specifications: Medium Access Control (MAC) Enhancements for Quality of Service (QoS), IEEE, June 2003.

[5] Y. Andreopoulos, N. Mastronarde, and M. van der Schaar, "CrossLayer Optimized Video Streaming over Wireless Multi-Hop Mesh Networks," IEEE J. Selected Areas in Comm., vol. 24, no. 11, pp. 1215-2104, Nov. 2006.

[6] W. Wei and A. Zakhor, "Multipath Unicast and Multicast Video Communication over Wireless Ad Hoc Networks," Proc. First Ann. Int'l Conf. Broadband Networks (Broadnets '04), pp. 496-505, 2004.

[7] J.G. Apostolopoulos, "Reliable Video Communication over Lossy Packet Networks Using Multiple State Encoding and Path Diversity," Proc. SPIE Visual Comm. and Image Processing (VCIP '01), Jan. 2001.

[8] J. Apostolopoulos, T. Wong, W. Tan, and S. Wee, "On Multiple Description Streaming with Content Delivery Networks," Proc. IEEE INFOCOM, 2002.

[9] S. Jain, M. Demmer, R. Patra, and K. Fall, "Using Redundancy to Cope with Failures in a Delay Tolerant Network," Proc. ACM Ann. Conf. Applications, Technologies, Architectures, and Protocols for Computer Comm. (SIGCOMM '05), Aug. 2005.

[10] R. Puri, K.-W. Lee, K. Ramchandran, and V. Bharghavan, "Forward Error Correction (FEC) Codes Based Multiple Description Coding for Internet Video Streaming and Multicast," Signal Processing: Image Communication, vol. 16, no. 8, pp. 745-762, May 2001.

[11] M. Kodialam and T. Nandagopal, "Characterizing Achievable Rates in Multi-Hop Wireless Networks: The Joint Routing and Scheduling Problem," Proc. ACM Int'l Conf. Mobile Computing and Networking, pp. 42-54, 2003.

[12] D. Krishnaswamy and J. Vicente, "Scalable Adaptive Wireless Networks for Multimedia in the Proactive Enterprise," Intel Technology J., vol. 8, no. 4, Nov. 2004.

[13] L. Xiao, M. Johansson, and S.P. Boyd, "Simultaneous Routing and Resource Allocation via Dual Decomposition," IEEE Trans. Comm., vol. 52, no. 7, pp. 1136-1144, July 2004.

[14] S. Roy, M. Covell, J. Ankcorn, S. Wee, and T. Yoshimura, "A System Architecture for Managing Mobile Streaming Media Services," Proc. IEEE Distributed Computing Systems Workshop, pp. 408-413, 2003.

[15] D. Singer, W. Belknap, and G. Franceschini, ISO Media File Format Specification - MP4 Technology under Consideration for ISO/IEC 14496-1:2002 Amd 3, Committee Draft, ISO/IEC JTC1/SC29/ WG11 MPEG01/N4270-1, July 2001.

[16] J.-R. Ohm, "Advances in Scalable Video Coding," Proc. IEEE, vol. 93, pp. 42-56, 2005

[17] M. van der Schaar and D.S. Turaga, "Cross-Layer Packetization and Retransmission Strategies for Delay-Sensitive Wireless Multimedia Transmission," IEEE Trans. Multimedia, vol. 9, no. 1, pp. 185-197, Jan. 2007.

[18] M. van der Schaar, S. Krishnamachari, S. Choi, and X. Xu, "Adaptive Cross-Layer Protection Strategies for Robust Scalable Video Transmission over 802.11 WLANs," IEEE J. Selected Areas in Comm., vol. 21, no. 10, pp. 1752-1763, Dec. 2003.

[19] D. Krishnaswamy, "Network-Assisted Link Adaptation with Power Control and Channel Reassignment in Wireless Networks," Proc. 3G Wireless Conf., pp. 165-170, 2002.

[20] K.-B. Song and S.A. Mujtaba, "On the Code-Diversity Performance of Bit-Interleaved Coded OFDM in Frequency-Selective Fading Channels," Proc. IEEE Vehicular Technology Conf. (VTC '03), vol. 1, pp. 572-576, 2003.

[21] P.A. Chou and Z. Miao, "Rate-Distortion Optimized Streaming of Packetized Media," Technical Report MSR-TR-2001-35, Microsoft Research, Feb. 2001.

[22] D. Bertsekas and R. Gallager, Data Networks. Prentice Hall, 1992.

[23] Y. Andreopoulos, A. Munteanu, J. Barbarien, M. van der Schaar, J. Cornelis, and P. Schelkens, "In-Band Motion Compensated Temporal Filtering," Signal Processing: Image Communication, special issue on subband/wavelet interframe video coding, vol. 19, no. 7, pp. 653-673, Aug. 2004.

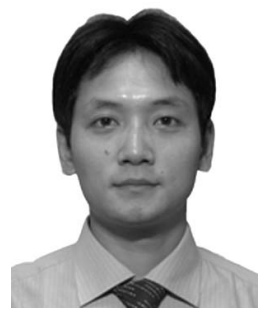

Xiaolin Tong received the master's degree in electrical engineering from the University of California, Los Angeles in September 2006. He obtained his bachelor's and master's degrees in computer science from the Beijing University of Posts and Telecommunications and the Institute of Computing Technology, Chinese Academy of Sciences, in 2002 and 2005, respectively. He is now with the Corporate R\&D Department, Qualcomm Inc.

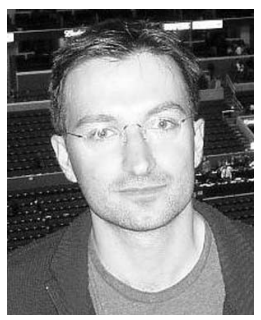

Yiannis Andreopoulos received the electrical engineering diploma and the MSc degree in signal processing systems from the University of Patras, Greece, in 1999 and 2000, respectively, and the $\mathrm{PhD}$ degree in applied sciences from the University of Brussels, Belgium, in May 2005, where he defended a thesis on scalable video coding and complexity modeling for multimedia systems. During his thesis work, he participated and was supported by the project MASCOT, a European Union Future and Emerging Technologies (FET) project. During his postdoctoral work at the University of California, Los Angeles, he performed research on cross-layer optimization of wireless media systems, video streaming, and theoretical aspects of rate-distortioncomplexity modeling for multimedia systems. Since October 2006, he has been a lecturer at the Queen Mary University of London, United Kingdom. During 2002-2003, he made several decisive contributions to the ISO/IEC JTC1/SC29/WG11 (Motion Picture Experts Group (MPEG)) committee in the early exploration on scalable video coding, which has now moved into the standardization phase. In 2007, he received the Most Cited Paper Award from Signal Processing: Image Communication. $\mathrm{He}$ is a member of the IEEE.

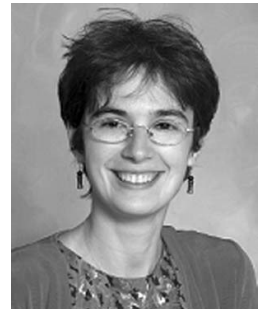

Mihaela van der Schaar received the $\mathrm{PhD}$ degree from the Eindhoven University of Technology, the Netherlands, in 2001. She is currently an associate professor in the Electrical Engineering Department at the University of California, Los Angeles. She has been an active participant to the ISO Motion Picture Expert Group (MPEG) standard since 1999, to which she made more than 50 contributions and for which she received three ISO recognition awards. She was also elected as a member of the Technical Committee on Multimedia Signal Processing and of the Technical Committee on Image and Multiple Dimensional Signal Processing, both of the IEEE Signal Processing Society. She was an associate editor of the IEEE Transactions on Multimedia and SPIE Electronic Imaging Journal. Currently, she is an associate editor of IEEE Transactions on Circuits and System for Video Technology and IEEE Signal Processing Letters. She holds 28 granted US patents and has several more pending. She received the US National Science Foundation Faculty Early Career Development (CAREER) Award in 2004, the IBM Faculty Award in 2005, the Okawa Foundation Award in 2006, the Best IEEE Transactions on Circuits and Systems for Video Technology Paper Award in 2005, and the Most Cited Paper Award from Signal Processing: Image Communication between 2004 and 2006. She is also the editor (with Phil Chou) of the book Multimedia over IP and Wireless Networks: Compression, Networking, and Systems. She is a senior member of the IEEE.

$\triangleright$ For more information on this or any other computing topic, please visit our Digital Library at www.computer.org/publications/dlib. 